ISSN: 0514-7336

DOI: https://doi.org/10.14201/zephyrus20167887110

\title{
REGISTRO PARA LA CUANTIFICACIÓN DE CERÁMICA ARQUEOLÓGICA: ESTADO DE LA CUESTIÓN Y UNA NUEVA PROPUESTA. PROTOCOLO DE SEVILLA (PRCS/14)
}

\section{Record for the quantification of archaeological pottery: state of art and a new proposal. Seville Protocol (PRCS/14)}

Andrés María Adroher Auroux*, César Carreras Monfort**, Rui de Almeida***, Adolfo Fernández Fernández ${ }^{* * * *}$, Jaime Molina Vidal ${ }^{* * * * *}$ y Catarina VIegas ${ }^{* * * * * *}$

* Dpto. de Prehistoria y Arqueología. Facultad de Filosofía y Letras. Campus Universitario de Cartuja. 18071 Granada.Correo-e: aadroher@ugr.es

** Dpto. de Ciencias de la Antigüedad. Edificio B. Facultad de Filosofía y Letras-UAB. 08193 Bellaterra (Barcelona). Correo-e: cesar.carreras@uab.cat

*** Investigador asociado a UNIARQ-Centro de Arqueologia da Univ. de Lisboa. Cl Dorada, 17 bajo. 21100 Punta Umbria (Huelva)

**** Talentia postdoc Research Contract. Program, Marie Skłodowska-Curie actions. Edificio Olga Gallego, despacho 28. Campus de As Lagoas. 32004 Orense. Correo-e: adolfo@uvigo.es

***** Dpto. de Prehistoria, Arqueología, H. ${ }^{a}$ Antigua y Filologías Griega y Latina. Facultad de Filosofía y Letras. Campus de San Vicente del Raspeig, s/n. 03690 San Vicente del Raspeig (Alicante). Correo-e: jaime. molina@ua.es

****** UNIARQ-Centro de Arqueologia da Univ. de Lisboa. Facultad de Letras. Univ. de Lisboa. Alameda da Universidade.1600-214 Lisboa (Portugal).Correo-e: c.viegas@letras.ulisboa.pt

Recepción: 16/05/2016; Revisión: 16/07/2016; Aceptación: 19/09/2016

Resumen: Presentamos una propuesta de cuantificación en ceramología protohistórica y clásica así como de representación de los resultados de dichas analíticas. Nuestro principal objetivo consiste en homogeneizar modelos de diagnóstico, análisis y publicación de los datos obtenidos de estudios de materiales cerámicos independientemente de su naturaleza, puesto que puede ser útil tanto de conjuntos estratigráficos como de series específicas. Consideramos que, de esta forma, se mejorará la interconexión no sólo de datos de unas zonas con otras, sino también de unos yacimientos con otros; además, resultará posible obtener resultados territorialmente más sólidos y contrastables que los que podemos obtener hasta este momento. Finalmente, a través del uso de ciertas convenciones en el análisis de la cerámica, podremos construir un aparato interpretativo de alto rango, que incorpore aspectos de carácter comercial, social, cultural e incluso simbólico.

Palabras clave: procedimiento; cálculo; vajilla; Península Ibérica; Protohistoria; romano.

Aвstract: We introduce here a new quantification proposal in protohistoric and classical pottery studies. The proposal also includes the way of representation of such analytics with the aim of making homogeneous 
diagnostic, analysis and publication models of any kind of ceramic material disregarding their nature. Therefore, it can be useful for either stratigraphy assemblages or particular series. This standard attempts to improve data interconnection between different areas, between different sites, so more solid and comparable values will be obtained at territorial level. At least, much better than the ones employed nowadays. Finally, using certain conventions in the pottery analysis will help us to build a high rank interpretative system, which may include aspects of commercial, social, cultural and, even, symbolic character.

Key words: procedure; calculation; ware; Iberian Peninsula; Protohistory; Roman.

\section{Introducción}

La cuantificación de cerámicas arqueológicas aún no ha impregnado los estudios técnicos y metodológicos entre investigadores de la Península Ibérica, siendo aún muy escasos los ejemplos de trabajos publicados sobre cerámicas que incluyan algún tipo de análisis estadístico de estas características. A ello añadimos la gran variedad y multiplicidad de formas de registro y técnicas de conteo, así como la diversidad de sus correspondientes aplicaciones y representaciones gráficas, lo cual ha impedido que puedan desarrollarse comparaciones entre conjuntos y excavaciones, por lo que, conforme transcurre el tiempo y se desarrollan más y más métodos de análisis e interpretación en la disciplina, se hace por momentos más evidente la necesidad de crear algún tipo de estándar, procedimiento o protocolo que permita una mayor comunicabilidad en la comparativa de resultados de un lugar a otro. Y más aún en el ámbito de la ceramología, al ser los elementos vasculares en arcilla los más frecuentes entre las evidencias arqueológicas desde su invención en el Neolítico.

En 2014, dentro del proyecto Amphorae ex Hispania (DGYCIT-HAR2011-28244) se propusieron unas sesiones comunitarias en Sevilla con el título Cuantificación de ánforas: protocolos y comparativas, de las que surgió una mesa de trabajo cuyo objetivo era desarrollar una propuesta de registro y análisis básico de materiales cerámicos en arqueología protohistórica y clásica. La finalidad prioritaria consistía en superar las dificultades y lagunas que se detectaban en la investigación desarrollada, principalmente, desde la Península Ibérica. Esta mesa se volvió a reunir el 17 de abril de 2015, aprovechando la hospitalidad del Instituto Arqueológico Alemán

(C) Universidad de Salamanca de Madrid, y consensuó un documento denominado Protocolo de Registro para la Cuantificación de Sevilla 2014 (PRCS/14), que aquí presentamos.

El principal objetivo consiste en llamar la atención sobre la necesidad de establecer estándares de valoración, clasificación, cuantificación y representación gráfica de resultados para los casos de materiales cerámicos extraídos de contextos arqueológicos, con la función de permitir una mayor comunicabilidad entre resultados de zonas distintas $y$, de esta forma, poder establecer conclusiones que superen las particularidades de un contexto o de un yacimiento, hasta alcanzar comportamientos en la cerámica en amplios territorios que nos permitan un mayor acercamiento a problemas relacionados con el comercio, las transformaciones culturales, comportamientos simbólicos e incluso evoluciones tecnológicas en la relación entre diversos grupos culturales, lo cual solamente puede hacerse si se establecen protocolos comunes de análisis que permitan esta mapificación macroterritorial.

\section{La cuantificación: de las primeras experiencias a las grandes escuelas}

Los intentos por establecer un único método de cuantificación han sido habituales al mismo ritmo que se desarrollaban los estudios en ceramología arqueológica y, especialmente, en la segunda mitad del s. xx. Sin embargo, la experiencia ha sido infructuosa debido a que se han desarrollado diferentes métodos, algunos de ellos compatibles y casi todos adecuados, cuya aplicabilidad varía en función de distintas variables -contexto, tipo de material, cronología, área excavada, entre otros-, por lo que, frecuentemente, resulta necesario combinarlos (Orton 
et al., 1993: 172; Arcelin y Tuffreau-Libre, 1998; Verdan, 2011).

Comúnmente, para los vasos de menor tamaño, agrupados en las categorías de cerámicas finas o comunes, la cuantificación se centra en fragmentos diagnósticos de bordes y bases por tener una mejor representatividad. Por el contrario, los grandes contenedores, especialmente los de carácter anfórico, presentan una proporción de fragmentos diagnósticos menor, que decrece hasta un $10 \%$ de su superficie total, por lo que se suelen emplear otros métodos que consideren fragmentos informes no diagnósticos. Esta es la razón principal por la que es necesario y compatible el uso de distintas técnicas de cuantificación. Hagamos, pues, una breve recapitulación sobre las numerosas y diversas experiencias que se han desarrollado en los estudios de cerámicas desarrolladas durante las últimas décadas con el objetivo de ilustrar el heterogéneo panorama existente.

La cuantificación es una técnica más de las que incorpora la New Archaeology o escuelas procesualistas en los años 70, de modo que la medición de los objetos, el empleo de la estadística y el estudio de las distribuciones requerirán el uso del número como técnica analítica (Orton, 1982), pero quizás de las más importantes aportaciones de las arqueologías procesualistas, ya que la cuantificación y la estadística forman parte del lenguaje matemático, y como se defendía desde los inicios del uso de estas técnicas no solo los ordenadores, sino que las mismas matemáticas construyen un "sistema abstracto de relaciones" (Shennan, 1992: 19, con abundante bibliografía sobre el tema) que permite finalmente un mejor y más preciso conocimiento del registro arqueológico. Y el éxito del uso de la estadística en el ámbito arqueológico no dependió solamente del desarrollo de la microinformática, que ayudó a poner al alcance de todos una serie de análisis hasta ese momento alcanzable solamente a unos pocos, sino que desde el momento en que en la arqueología decidimos hacer algo más que recoger algunos objetos más o menos valiosos procedentes del pasado y consideramos la posibilidad de recoger todo lo que encontrábamos en nuestros trabajos de campo, se hizo imprescindible el uso de técnicas

(c) Universidad de Salamanca que nos ayudaran a gestionar tal cantidad de información, para generar datos que permitieran interpretar una gran cantidad de analíticas y de esa forma establecer normas de comportamiento que permitan interpretar esos datos arqueológicos en clave histórica, cual es el verdadero objetivo de nuestra disciplina.

Un origen reciente de la cuantificación cerámica ha de rastrearse en las excavaciones desarrolladas en Cartago por la University of Michigan en la década de 1970, dirigidas por J. H. Humphrey. El método, establecido entre J. W. Hayes y J. A. Riley aunque normalizado y publicado por este último autor (1976: 125-131), incluía desde el registro en campo hasta la publicación de las tablas de resultados. Se primaba la contabilización e identificación de todos los fragmentos -bordes, bases, asas e informes-, incluyendo peso desglosado según tipología y porcentajes de todas las variables (Fig. 1). Este sistema de registro fue el utilizado en la publicación de las siguientes campañas, si bien fue reducido en su publicación para facilitar la síntesis y comprensión, representando el número total de fragmentos - para cada tipo de cerámica-, su peso y los porcentajes de ambas variables (Tomber, 1989). A partir de esta experiencia los análisis cuantitativos de cerámicas emprendieron un fructífero desarrollo en el que la adaptación a diferentes tipos y clases cerámicas no era fácil. Especialmente significativo es el caso de las grandes vasijas (almacenamiento o ánforas), con una mayor presencia de fragmentos informes que obligan a acometer estrategias diversas. De ahí que se hayan desarrollado dos ámbitos preferentes de cuantificación (Pollard, 1990) que cualquier protocolo de registro debe contemplar: el peso-PFR: peso de fragmentos- y el recuento de fragmentos -NFR: número de fragmentos-.

El peso de fragmentos - PFR - en sus distintas variantes es un método muy utilizado, sobre todo en el ámbito anglosajón (Tomber, 1993: 150), que consigue obviar el diferente grado de fragmentación, considerando grandes volúmenes de material informe y adaptándose perfectamente a yacimientos con registros cerámicos poco abundantes. Es un método que requiere un elevado grado de conocimientos

Zephyrus, LXXVIII, julio-diciembre 2016, 87-110 


\begin{tabular}{|c|c|c|c|c|c|c|c|c|c|c|}
\hline \multicolumn{11}{|c|}{ DEPOSIT XX $\quad\left(P_{3} F-L\right.$ 200) } \\
\hline Type & $\begin{array}{l}\text { No. of } \\
\text { Rims }\end{array}$ & $\begin{array}{l}\text { No. of } \\
\text { Bases }\end{array}$ & $\begin{array}{c}\text { No. of } \\
\text { Handles }\end{array}$ & $\begin{array}{c}\text { No. of } \\
\text { Handles }\end{array}$ & $\begin{array}{l}\text { Percent } \\
\text { No. RBHS }\end{array}$ & $\begin{array}{l}\text { Wt. of } \\
\text { Rims }\end{array}$ & $\begin{array}{l}\text { Wt. of } \\
\text { Bases }\end{array}$ & $\begin{array}{c}\text { Wt. of } \\
\text { Handles }\end{array}$ & $\begin{array}{l}\text { Wt. of } \\
\text { Sherds }\end{array}$ & $\begin{array}{c}\text { Percent } \\
\text { Wt. RBHS }\end{array}$ \\
\hline Black Glaze & 13 & 4 & 0 & 25 & 1.0 & 65 & 35 & 0 & 60 & 0.2 \\
\hline Arretine & 7 & 7 & 0 & 18 & 0.8 & 32 & 60 & 0 & 90 & 0.2 \\
\hline Imitation T.S. & 4 & 0 & 0 & 1 & 0.1 & 15 & 0 & 0 & 5 & 0.0 \\
\hline S. Gaulish & 0 & 0 & 0 & 1 & 0.0 & 0 & 0 & 0 & 5 & 0.0 \\
\hline Misc. Red Glos & 0 & 2 & 0 & 3 & 0.1 & 0 & 30 & 0 & 7 & 0.0 \\
\hline Pompeian Red & 1 & 0 & 0 & 0 & 0.0 & 5 & 0 & 0 & 0 & 0.0 \\
\hline African Red Slip & 59 & 13 & 2 & 107 & 4.5 & 740 & 160 & 45 & 65 & 1.3 \\
\hline Misc. ARS Lamps & 0 & 0 & 0 & 8 & 0.2 & 0 & 0 & 0 & 73 & 0.1 \\
\hline ARS Lamp Type II & 0 & 0 & 0 & 1 & 0.0 & 0 & 0 & 0 & 20 & 0.0 \\
\hline E. Roman Lamps & 0 & 0 & 0 & 19 & 0.5 & 0 & 0 & 0 & 50 & 0.1 \\
\hline Misc. Buff Lamps & 0 & 0 & 0 & 6 & 0.1 & 0 & 0 & 0 & 30 & 0.0 \\
\hline ARS Black Top & 12 & 2 & 1 & 51 & 1.6 & 192 & 50 & 5 & 400 & 0.8 \\
\hline Post Toman Glaze & 2 & 0 & 0 & 11 & 0.3 & 15 & 0 & 0 & 55 & 0.1 \\
\hline Misc. Burnisched & 0 & 1 & 0 & 1 & 0.0 & 0 & 5 & 0 & 5 & 0.0 \\
\hline Misc. Amphoras & 15 & 7 & 30 & 2168 & 55.5 & 1440 & 1180 & 3310 & 57215 & 78.5 \\
\hline E.R. Amphora 5 & 1 & 0 & 1 & 16 & 0.4 & 30 & 0 & 515 & 810 & 1.7 \\
\hline E.R. Amphora 6 & 0 & 0 & 0 & 3 & 0.1 & 0 & 0 & 0 & 120 & 0.1 \\
\hline L.R. Amphora 3 & 0 & 0 & 1 & 21 & 0.5 & 0 & 0 & 30 & 195 & 0.3 \\
\hline L.R. Amphora 4 & 1 & 0 & 1 & 26 & 0.7 & 30 & 0 & 140 & 790 & 1.2 \\
\hline L.R. Amphora 5 & 2 & 0 & 0 & 10 & 0.3 & 135 & 0 & 0 & 115 & 0.3 \\
\hline L.R. Amphora 6 & 0 & 0 & 0 & 1 & 0.0 & 0 & 0 & 0 & 5 & 0.0 \\
\hline L.R. Amphora 7 & 0 & 0 & 0 & 3 & 0.1 & 0 & 0 & 0 & 60 & 0.1 \\
\hline Grooved Amphora & 0 & 0 & 0 & 1 & 0.0 & 0 & 0 & 0 & 25 & 0.0 \\
\hline Misc. Cook Ware & 59 & 5 & 8 & 231 & 7.6 & 595 & 160 & 135 & 1500 & 3.0 \\
\hline Coarse Gritted & 6 & 0 & 0 & 0 & 0.1 & 115 & 0 & 0 & 0 & 0.1 \\
\hline E.R. Coorware I & 5 & 0 & 0 & 13 & 0.4 & 80 & 0 & 0 & 90 & 0.2 \\
\hline L.R. Cookware I & 14 & 0 & 0 & 4 & 0.4 & 155 & 0 & 0 & 45 & 0.2 \\
\hline L.R. Cookware II & 1 & 0 & 0 & 0 & 0.0 & 10 & 0 & 0 & 0 & 0.0 \\
\hline L.R. Cookware IV & 0 & 0 & 0 & 2 & 0.0 & 0 & 0 & 0 & 20 & 0.0 \\
\hline Plain Buff Wares & 90 & 24 & 35 & 455 & 15.1 & 2185 & 450 & 705 & 3220 & 8.2 \\
\hline Misc. Scraps & 0 & 0 & 0 & 142 & 3.5 & 0 & 0 & 0 & 715 & 0.9 \\
\hline Miasc. Coarse & 14 & 2 & 2 & 21 & 1.0 & 395 & 55 & 30 & 130 & 0.8 \\
\hline Vaulting Tubes & 0 & 0 & 0 & 128 & 3.2 & 0 & 0 & 0 & 825 & 1.0 \\
\hline Thin-Walled Ware & 5 & 6 & 3 & 28 & 1.0 & 10 & 35 & 10 & 36 & 0.1 \\
\hline Flanged Bowl 1 & 4 & 0 & 0 & 1 & 0.1 & 165 & 0 & 0 & 10 & 0.2 \\
\hline Buff Flanged Bowl & 2 & 0 & 0 & 0 & 0.0 & 5 & 0 & 0 & 0 & 0.0 \\
\hline Misc. Flanged Bowl & 1 & 0 & 0 & 0 & 0.0 & 35 & 0 & 0 & 0 & 0.0 \\
\hline Mortaria & 0 & 1 & 0 & 0 & 0.0 & 0 & 35 & 0 & 0 & 0.0 \\
\hline Totals & 318 & 74 & 84 & 3526 & 100.0 & 6452 & 2255 & 4925 & 66791 & 100.0 \\
\hline Total RBHS & & & & & & & & & & \\
\hline
\end{tabular}

Fig. 1. Tabla del depósito XX de Carthago, excavaciones de la Univ. de Michigan de 1976 (Hayes, 1978: 44, vectorizado por los autores a partir de los originales).

macroscópicos sobre pastas, engobes u otros elementos no estrictamente tipológicos. Además, la muestra que genera presenta un grado de incertidumbre mayor que si se consideraran sólo las partes que ofrecen una clasificación tipológica más fiable como los bordes. Asimismo, otorga una mayor representación a las cerámicas de mayor tamaño, paredes más gruesas o una densidad más elevada, lo que obliga a utilizar correctores modulares. Existen diversas variantes para contrarrestar algunos de estos problemas inherentes a este método como peso ajustado, corrector de superficie, desplazamiento por el agua y peso medio del vaso (Tomber, 1993; Orton et al., 1993).

$\mathrm{El}$ recuento de fragmentos $-N F R$ - es el sumatorio de perfiles completos, bordes, fondos, asas, decoraciones y amorfos. Presenta múltiples modalidades, 
ofreciendo una mayor comodidad y facilidad de aplicación, y el inconveniente de sobrevalorar los tipos que se fragmentan en un número mayor de partes o tratar de forma desigual algunas partes de las piezas, como las bases o las paredes sin forma, que presentan mayores dificultades de clasificación. Por eso es habitual que se contabilicen sólo fragmentos diagnosticables, por lo general asas, bases $y$, preferentemente, bordes, al ser la parte que proporciona una adscripción tipológica más fiable de la mayoría de las clases cerámicas. Este dato resulta interesante especialmente desde el punto de vista de la gestión museográfica, aunque puede ser utilizado como información complementaria para calcular otras variables, como los índices de erosión o los de fragmentación.

El panorama europeo de cuantificación cerámica es muy variado y desigual. En el ámbito anglosajón desde la década de 1980 se observa la incorporación de sistemas de registro doble $-N F R$ y PFR-, ofreciendo la información perfectamente tabulada por cantidades y porcentajes (Whitehouse et al., 1985) (Fig. 2A). A partir de entonces el doble registro ha sido el modo más habitual de documentar, calcular y presentar los conjuntos cerámicos.

En Italia (Carignani et al., 1986; Anselmino et al., 1986) con frecuencia se contabiliza una sola variable, habitualmente fragmentos totales o solo bordes, presentados en gráficos porcentuales difíciles de interpretar al no tener tablas de cuantificación, un error muy habitual.

En Francia, en torno a la escuela Aix/Marsella, se fue generando un sistema de cuantificación y de representación de los datos, que sigue en vigencia en la actualidad, consistente en registrar la mayor cantidad de variables posibles orientado preferentemente al cálculo del Número Mínimo de Individuos $-N M I-$. Se inicia así una corriente de cuantificación ligada al sistema de registro conocido como SYSLAT, y que vemos culminado en el Protocolo de Beuvray (Arcelin y Tuffreau-Libre, 1998).

No ha sido tan habitual hallar datos cuantificados en los trabajos centroeuropeos y cuando lo hacen se inclinan mayoritariamente hacia los parámetros típicos de la escuela anglosajona: fragmentos, peso y sus porcentajes. Destaca un reciente ejemplo de la Universidad de Leuven (Bélgica) sobre las ánforas del yacimiento turco de Sagalassos (Corremans et al., 2010), o en el caso del proyecto ICRATES que valoriza la cuantificación como herramienta para el estudio de la historia económica (Bes y Poblome, 2008).

Será precisamente el Protocolo de Beuvray el que incorpore aspectos como el Número Tipológico de Individuos -NTI- o el Número Máximo de Individuos, extraído de la cuantificación a partir de los fragmentos de bordes y bases distintos de una misma tipología que permanecen después de intentar cualquier tipo de unión. No obstante, sin duda alguna lo que más se potencia desde esta escuela es el $N \dot{u}$ mero Minimo de Individuos -NMI- sobre cualquier otra variable como el método más indicado para cuantificar cerámica. A partir de una selección del material cerámico - piezas completas, bordes, pivotes y asas-, se agrupan y asignan los fragmentos que no encajan pertenecientes al mismo individuo/tipo y se le asigna el valor más elevado obtenido sobre las diferentes partes morfológicas. En muestras con un número de fragmentos elevado se acepta realizar tan sólo el NMI de los bordes, al cual denominaremos NMIb para diferenciarlo del anterior ponderado. En los casos en los que hay varios fragmentos de un tipo pero el NMI es 0 , se acepta ponderarlo colocando un 1. El NMI es una estimación del mínimo número de ejemplares o cerámicas completas que representan los fragmentos recuperados (Orton et al., 1993: 172; Arcelin y Tuffreau-Libre, 1998), constatando de esa forma el mínimo número de piezas/vasijas/ individuos que se han conservado en el conjunto.

De forma complementaria otros investigadores han utilizado la estimación de Equivalentes de Vasija $-E V E-$, que define cada fragmento como una porción de la vasija completa, aunque por motivos prácticos se suele limitar a contabilizar la proporción en bases y bordes, sumando ambas variables y dividiendo el resultado por dos; este resultado se divide entre 100 para obtener en unidades los vasos estimados finales. Por ello, con frecuencia, se reduce al denominado equivalente de borde (Orton, 1982: 164-167), calculado a partir del porcentaje del fragmento respecto a 


\begin{tabular}{|c|c|c|c|c|c|c|c|c|}
\hline \multicolumn{9}{|c|}{ Fabrics 10-24 (mostly amphorae) consisted of: } \\
\hline \multirow[t]{2}{*}{ Fabric } & \multicolumn{4}{|c|}{ Quantity } & \multicolumn{4}{|c|}{ Weight } \\
\hline & Sherds & $\%$ & sherds & $\%$ & Kg. & $\%$ & Kg. & $\%$ \\
\hline \multicolumn{9}{|l|}{ North African } \\
\hline 11. Many inclusions & 1 & 0.3 & 133 & $40-7$ & 0.05 & $\begin{array}{c}57.6 \\
0.6\end{array}$ & 4.60 & 58.2 \\
\hline \multicolumn{9}{|l|}{ Other } \\
\hline 13. LRA 3 & 95 & 29.1 & & & 0.85 & 10.8 & & \\
\hline 14. & 40 & 12.2 & & & 0.90 & 11.4 & & \\
\hline 15. & 12 & 3.7 & & & 0.30 & 3.8 & & \\
\hline 15.(a) & 2 & 0.6 & & & 0.10 & 1.3 & & \\
\hline 16. LRA 4 & 31 & 9.5 & & & 0.75 & 9.5 & & \\
\hline 18. LRA 5 & 5 & 1.5 & & & 0.50 & 0.6 & & \\
\hline 19.(a) & 5 & 1.5 & & & 0.20 & 2.5 & & \\
\hline 20. & 1 & 0.3 & & & - & - & & \\
\hline 23. & 1 & 0.3 & & & 0.10 & 1.3 & & \\
\hline 24.(b). LRA 1 & 1 & 0.3 & & & - & - & & \\
\hline 24.(c). LRA2 & 1 & 0.3 & 194 & $59-3$ & 0.05 & 0.6 & 3.30 & 41.8 \\
\hline Total & & & 327 & $100-0$ & & & 7.90 & 100.0 \\
\hline
\end{tabular}

\begin{tabular}{|c|c|c|c|c|c|c|c|c|}
\hline \multicolumn{9}{|c|}{ Fase 1} \\
\hline categorie & Forme & $\pi$ & \begin{tabular}{l|l|}
$C$ & $B$ \\
\end{tabular} & $\mathrm{~F}$ & A & $\mathrm{P}$ & NMI & no \\
\hline \multirow{3}{*}{ amph.hispaniques } & Almagro 51A-B & 25 & & & 2 & 23 & 1 & 266 \\
\hline & Almagro 51C & 39 & & 1 & & 38 & 1 & 267 \\
\hline & Dressel 23 & 3 & 3 & & . & & 3 & $262-265$ \\
\hline \multirow{9}{*}{ amph. africaines } & Dressel 30 & 1 & 1 & & & & 1 & 268 \\
\hline & \begin{tabular}{|l|} 
Africaine 1 \\
\end{tabular} & 1 & 1 & & & & 1 & 269 \\
\hline & Spatheia & 46 & 3 & 11 | & 9 & 23 & 11 & $270-277$ \\
\hline & Keay XXV & 2 & 2 & & & & 2 & 278 \\
\hline & Keay XXXVB & 15 & 3 & & 2 & 10 & 3 & 279,284 \\
\hline & Keay XXXVI & 1 & 1 & & & & 1 & 285 \\
\hline & Keay XC? & 1 & 1 & & & & 1 & 283 \\
\hline & Keay VIIIB & 4 & 4 & & . & & 4 & $280-282$ \\
\hline & cylindrique indetérminée & 822 & 3 & 9 & 13 & 797 & 9 & $286-289$ \\
\hline \multirow{7}{*}{ amph, orientales } & LRA 1 & 1210 & \begin{tabular}{|l|l|}
2 & 21 \\
\end{tabular} & 10 & 22 & 1155 & 23 & $290-299$ \\
\hline & LRA 2 & \begin{tabular}{|c|}
1 \\
\end{tabular} & & & 1 & & 1 & 308 \\
\hline & LRA 3 & 264 & 1 & 13 & 10 & 240 & 13 & $300-303$ \\
\hline & LRA 4 & 119 & 6 & & 4 & 109 & 6 & $304-306$ \\
\hline & \begin{tabular}{|l|} 
LRA 5 \\
\end{tabular} & 42 & 1 & & 2 & 39 & 1 & 307 \\
\hline & Agora M273 & 85 & 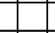 & 4 & & 80 & 3 & $309-311$ \\
\hline & Agora M334 & 1 & & 1 & & & 1 & 312 \\
\hline amph. italiques & Keay LII & 111 & 9 & 1 & 6 & 95 & 9 & $313,314,316$ \\
\hline \multirow{4}{*}{ origine indeterminée } & Inét. (pâte micacée) & 26 & & & 1 & 25 & 1 & \\
\hline & Bonifay-Villedieu 1989, fig. 14, no 18 & 2 & 1 & & 1 & & 1 & 315 \\
\hline & Bonifay-Villedieu 1989, fig. 14, no 15 Vila-Roma 8/18 & 1 & 1 & & & & 1 & 317 \\
\hline & Indéterminée & 825 & 6 & 9 & 18 & 793 & 9 & \\
\hline TOTAL & & 3648 & & & & & 107 & \\
\hline \multicolumn{9}{|c|}{ Fase 2} \\
\hline categorie & Forme & $\pi$ & B & $\mathrm{F}$ & A & $P$ & NMI & no \\
\hline \begin{tabular}{|l|} 
amph.hispaniques \\
\end{tabular} & indéterminée & 4 & & & & 4 & 1 & \\
\hline \multirow{5}{*}{ amph. africaines } & spatheion & 26 & 1 & & 1 & 24 & 1 & 318 \\
\hline & Keay XL & 1 & 1 & & & & 1 & 321 \\
\hline & Keay VLVII & 1 & 1 & & 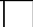 & & 1 & 319 \\
\hline & Albenga 11/12 & 1 & 1 & & 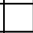 & & 1 & 320 \\
\hline & cylindrique indetérminée & 211 & & 5 & 4 & 202 & 5 & $322-324$ \\
\hline \multirow{3}{*}{ amph. orientales } & LRA 1 & 24 & 2 & & 4 & 18 & 2 & 326 \\
\hline & LRA 3 & 5 & & & & 5 & 1 & \\
\hline & \begin{tabular}{|l|} 
LRA 4 \\
\end{tabular} & 2 & 1 & & & 1 & 1 & 325 \\
\hline \multirow[t]{2}{*}{ amph. italiques } & Keay LII & 9 & & & 2 & 7 & 1 & \\
\hline & Indéterminée & 139 & & 1 & & 138 & 1 & \\
\hline TOTAL & & 423 & & & & & 16 & \\
\hline
\end{tabular}

FIG. 2. A) Excavaciones en la Schola Praecorum II (Whitehouse et al., 1985: 178); B) Marsella. Puits de la rue Bon-Jésus (Bonifay et al., 1998: 407, tabl. LXIX, vectorizado por los autores a partir de los originales). los $360^{\circ}$ de un ejemplar completo, con la ayuda de plantillas de radio. Este es el método por el que C. Orton parece decantarse tras la realización de distintas simulaciones por ser el que proporcionaba mejores resultados (Orton et al., 1993: 172). La diferencia respecto al NMI es que no se intenta que los fragmentos se unan, sino tan sólo sumar los porcentajes de todos los fragmentos de la misma tipología. De este modo se ahorra tiempo y se supera el problema derivado del distinto grado de ruptura de los tipos y clases cerámicas, aunque sólo es aplicable a investigaciones directas y no a las ya publicadas, que generalmente sólo ofrecen el número bruto de fragmentos.

Adicionalmente también se puede realizar un tipo de recuento basado en el EVE Corregido con un coeficiente fijo de fragmentación denominado Módulo de Ruptura-MR- (Mateo y Molina, 2016). Cada tipo cerámico en función de su forma y resistencia presenta patrones de fragmentación similares que son susceptibles de ser calculados estadísticamente. La confección de las tablas de Módulo de Ruptura para cada clase y tipo cerámico, hasta ahora sólo calculadas para las ánforas, permite cuantificar de forma automática el Eve de borde de un conjunto cerámico de forma altamente fiable, agilizando la recogida de datos, permitiendo aplicarlo a investigaciones ya publicadas siempre que se haya publicado el número bruto de fragmentos. 
A pesar de la existencia de dos grandes escuelas, finalmente la aplicación de los sistemas de cuantificación es muy heterogénea, a lo que habría que añadir la forma dispar de calcular algunas variables, especialmente el NMI, lo que genera auténticos artificios cuando queremos establecer comparaciones. Esta falta de homogeneidad en los planteamientos $y$, sobre todo, en su aplicación es el problema central de la cuantificación cerámica ya que impide comparar los datos de distintos yacimientos, obstaculizando los análisis ulteriores de carácter económico, social o cultural macroterritoriales.

En la actualidad, a pesar de esta oferta de técnicas de cuantificación, un porcentaje muy elevado de publicaciones sigue sin tener en cuenta estas necesidades de la cuantificación en Arqueología en general y en los estudios cerámicos en particular para permitir la universalización y contrastación de los datos y construir discursos supraterritoriales; y en otros casos nos encontramos con que se utilizan pero de modo inadecuado por falta de una formación correcta en su aplicación real. Ello ha llevado al error habitual de tratar de comparar conjuntos diferentes combinando los datos porcentuales sin tener en cuenta que provienen de analíticas distintas. Sirva como recurrente ejemplo la comparación entre datos resultantes de contextos publicados de la misión italiana -a partir de fragmentos-, británica y americana - pesosen Cartago (Anselmino et al., 1986). Más recientes son los esfuerzos de S. J. Keay por comparar contextos de diferentes yacimientos del Mediterráneo (1998: 152-153) o los de P. Reynolds en su obra sobre el comercio en este mismo contexto (2010: tables) y que ilustran perfectamente estas dificultades (Fig. 3).

La utilización de datos porcentuales es necesaria, pero su interpretación puede conllevar pérdida de información o generar correlaciones ficticias si no se consideran variables como el origen, el contenido o la capacidad de los envases. Los porcentajes son útiles en ámbitos con similares condiciones de producción y cronología, porque si hay producción local las importaciones siempre tendrán un porcentaje menor o si las ocupaciones tienen diferentes cronologías, los asentamientos con una ocupación larga tenderán a presentar valores porcentuales inferiores. En definitiva,

\begin{tabular}{|c|c|c|c|c|c|}
\hline $\begin{array}{l}\text { Date } \\
\text { AD 420/440 }\end{array}$ & African & Eastern & Other & F. Ombelicato & F. No. sherds/MNI \\
\hline Rome (Magna Mater: Saggio I-L) & $41.5 \%$ & $22 \%$ & $36.5 \%$ & $0 \%$ & 401 sherds or MNI \\
\hline $\begin{array}{l}\text { Porto Torres (couche iva. I) } \\
\text { AD 400/450 }\end{array}$ & $65.6 \%$ & $6.0 \%$ & $28.4 \%$ & $0 \%$ & 1080 sherds \\
\hline $\begin{array}{l}\text { Tarragona (Santa Tecla) } \\
\text { AD 440/450 }\end{array}$ & $17.7 \%$ & $22.2 \%$ & $59.8 \%$ & $0 \%$ & 45 sherds \\
\hline Carthage (Bristish) & $52.65 \%$ & $31.97 \%$ & $15.36 \%$ & $0 \%$ & $17.555 \mathrm{~kg}$ \\
\hline Tarragona (Vila-romà) & $24.5 \%$ & $25.5 \%$ & $50 \%$ & $0 \%$ & 27840 sherds \\
\hline Marseilles (Bourse Periode I) & $17.7 \%$ & $43.9 \%$ & $38.3 \%$ & $0 \%$ & MNI 107 \\
\hline Rome (Schola Praeconum I) & $42.5 \%$ & $27.4 \%$ & $30.1 \%$ & $0 \%$ & 9960 sherds \\
\hline Naples (CM III) & $57.1 \%$ & $7.1 \%$ & $35.8 \%$ & $0 \%$ & NA \\
\hline $\begin{array}{l}\text { Porto Torres (couche iv.b) } \\
\text { AD } \mathbf{4 4 0 / 4 8 0}\end{array}$ & $55.8 \%$ & $6 \%$ & $38.2 \%$ & $0 \%$ & 1303 sherds \\
\hline $\begin{array}{l}\text { Rome (Magna Mater: Saggio P) } \\
\text { After AD } \mathbf{4 6 0 / 4 7 0}\end{array}$ & $38.1 \%$ & $26.1 \%$ & $35.8 \%$ & $0 \%$ & 507 sherds or MNI \\
\hline $\begin{array}{l}\text { Turris Libisonis (couche v) } \\
\text { Late 5th AD }\end{array}$ & $52.9 \%$ & $19.3 \%$ & $27.8 \%$ & $0 \%$ & 272 sherds \\
\hline Carthage (Italian) & $39.1 \%$ & $19.4 \%$ & $15.4 \%$ & $0 \%$ & NA \\
\hline Carthage (British) & $51.57 \%$ & $24.69 \%$ & $23.72 \%$ & $0 \%$ & $131.582 \mathrm{~kg}$ \\
\hline Tarragona (Antiqua Audiència) & $24 \%$ & $34 \%$ & $42 \%$ & $0 \%$ & MNI 50 \\
\hline $\begin{array}{l}\text { Tarragona (Trinquet Vell) } \\
\text { AD } \mathbf{5 0 0}\end{array}$ & $33.3 \%$ & $26.8 \%$ & $39.7 \%$ & $0 \%$ & 93 sherds \\
\hline Carthage (British) & $47.37 \%$ & $21.92 \%$ & $30.69 \%$ & $0 \%$ & $232.818 \mathrm{~kg}$ \\
\hline $\begin{array}{l}\text { Naples (CM } 234 \text { \& 248) } \\
\text { AD 500/530 }\end{array}$ & $30 \%$ & $13.1 \%$ & $56.1 \%$ & $0 \%$ & $40.810 \mathrm{~kg}$ \\
\hline $\begin{array}{l}\text { Rome (Schola Praeconum II) } \\
\text { AD 525/535 }\end{array}$ & $40.5 \%$ & $40.8 \%$ & $18.7 \%$ & $0 \%$ & 1141 sherds \\
\hline $\begin{array}{l}\text { Carthage (British) } \\
\text { AD 525/530-580 }\end{array}$ & $57.20 \%$ & $26.53 \%$ & $16.26 \%$ & $0 \%$ & $165.060 \mathrm{~kg}$ \\
\hline $\begin{array}{l}\text { Alicante (Benalúa 42.4) } \\
\text { AD 55/575 }\end{array}$ & $39.84 \%$ & $21.05 \%$ & $39.04 \%$ & $0 \%$ & MNI $133(+)$ \\
\hline $\begin{array}{l}\text { Carthage (British) } \\
\text { AD 550/early 7th centuries AD }\end{array}$ & $63.80 \%$ & $11.27 \%$ & $24.92 \%$ & $0 \%$ & $89.972 \mathrm{~kg}$ \\
\hline $\begin{array}{l}\text { Cartagena (Calle Soledad) } \\
\text { AD 500/600 }\end{array}$ & $37.5 \%$ & $34.3 \%$ & $28 \%$ & $0 \%$ & 32 sherds \\
\hline $\begin{array}{l}\text { Tarragona (Torre Audiencia) } \\
\text { Late 6th/early 7th century AD }\end{array}$ & $75.25 \%$ & $12.6 \%$ & $12.15 \%$ & $0 \%$ & MNI 187 \\
\hline Carthage (British) & $53.28 \%$ & $21.39 \%$ & $25.31 \%$ & $0 \%$ & $16.988 \mathrm{~kg}$ \\
\hline Marseilles (Bourse: Periode 2) & $48.14 \%$ & $33.13 \%$ & $18.51 \%$ & $0 \%$ & MNI 54 \\
\hline Naples (CM) & $18.8 \%$ & $34.6 \%$ & $46.8 \%$ & $0 \%$ & 389 sherds \\
\hline S. Antonino (T.4-5) & $47.5 \%$ & $17.7 \%$ & $30 \%$ & $4.8 \%$ & MNI 50 \\
\hline $\begin{array}{l}\text { 7th century AD } \\
\text { Marseilles (Bourse: Periode 3) }\end{array}$ & $97 \%$ & $2 \%$ & $1 \%$ & $0 \%$ & MNI 18 \\
\hline $\begin{array}{l}\text { Early/mid /th century AD } \\
\text { S. Antonino }\end{array}$ & $60.2 \%$ & $11.2 \%$ & $13.5 \%$ & $15.1 \%$ & MNI 68 \\
\hline
\end{tabular}

FIG. 3. Ensayo de comparación entre contextos publicados (Keay, 1998: 153, appendix 1 , vectorizado por los autores a partir de los originales). 
el uso de porcentajes es necesario e ilustrativo pero se ha de ser cauto en la interpretación de sus resultados. En el caso de las ánforas, a partir de la conversión de los pesos o NMI en litros de productos transportados y el cálculo de densidades, se pueden comparar distintos tipos de forma aislada o comparar productos -vinos, aceite, garum-.

Si no se utilizan porcentajes, las comparaciones entre cantidades de distintos conjuntos deben tener en cuenta el contexto en donde se hallaron. Normalmente se establece la relación con el área excavada, es decir, con el volumen de tierra extraído del contexto donde aparece el material analizado (Carreras, 2000: 54-58), técnica utilizada anteriormente por Rice (1987: 289) y por Millett (1991: 238). El método es razonable, pero resulta complicado de aplicar a nivel práctico porque el cálculo de volúmenes de estratos no es siempre sencillo. En consecuencia, para simplificar puede ser válido - previa indicación de la proveniencia del datoutilizar la superficie en vez del volumen. Con el fin de conseguir establecer comparaciones de un mismo tipo se ha propuesto el cálculo de densidades a partir del peso total de fragmentos y de la cantidad de sedimento excavado estimado (Rice, 1987: 289), evitando así uno de los problemas de establecer comparaciones relativas mediante la utilización de porcentajes.

Otro de los problemas para establecer comparaciones es la dimensión temporal, pues algunos autores no indican el sistema tipológico y, por ende, cronológico, de referencia que se ha utilizado para la clasificación del material, lo que sería necesario para conocer la procedencia de dicho dato cronológico. A pesar de ello, se pueden utilizar otros sistemas para corregir esta descompensación posible, por ejemplo, dividiendo las cantidades por el número de años de cada periodo, pero los resultados pueden proporcionar valores extremos. Para resolverlo, es tan sólo recomendable comparar conjuntos aproximadamente de la misma cronología.

\section{La cuantificación en la Península Ibérica: viejos y nuevos caminos, perspectivas $y$ necesidades}

En la Península Ibérica hasta mediados de la década de 1970 no encontramos la aplicación de métodos de cuantificación cerámica. A pesar de ello, se detectan leves influencias de la New Archaeology y la escuela del pensamiento Procesual y su interés por "medir, pesar y contar", como es el caso de los primeros intentos de cuantificación en el Castro da Rutura en Portugal (Gonçalves, 1971) (Fig. 3a).

Entre la segunda mitad de la década de 1970 y mediados de los años 80 irrumpe la praxis de la cuantificación y el tratamiento estadístico de conjuntos cerámicos, todavía como una novedad metodológica, para convertirse en línea de trabajo recurrente sólo en la década de los años 90. En cualquier caso, las aplicaciones y resultados fueron muy desiguales mostrando una gran heterogeneidad metodológica, analítica y formal en la presentación e interpretación de los resultados. Cabría destacar el ámbito de la investigación anfórica que paulatinamente pasará de los tradicionales estudios de presencia y difusión de naturaleza estrictamente tipológica, a la cuantificación y análisis complejo que combinaba tipos y pastas.

Los primeros análisis cuantitativos modernos los hallamos en los volúmenes monográficos de las Fouilles de Conimbriga, donde se ensaya un intento de cuantificación de la terra sigillata sudgálica basado en el número de fragmentos con forma diagnosticable (Delgado et al., 1975: 70 y fig. 1), u otros grupos cerámicos publicando de forma individual el número de fragmentos de cada uno de los tipos representados (Alarcão, 1976).

Todavía en el ámbito lusitano en 1978 encontramos un estudio sobre el consumo de ánforas en la ciudad de Setúbal, en la cual se explica de forma textual que la cuantificación por tipos se realiza exclusivamente con base en "ejemplares enteros sumados a los fragmentos de borde". Se desglosan los valores parciales y se presenta la cuantificación total mediante un gráfico de barras y un cuadro cronotipológico (Coelho-Soares y Silva, 1978: 173 y figs. 2-3) (Fig. 4a). Habrá que esperar al estudio del Castelo de Alcácer do Sal (Silva et al., 1980-81) para encontrar una aplicación conjunta de métodos cuantitativo-estadísticos a cerámicas de la Edad del Hierro y romanas (Fig. 4c).

En Espańa se siguió la misma pauta, pero como la influencia de la "escuela francesa" del mundo clásico era menor las primeras aportaciones cuantificadas 


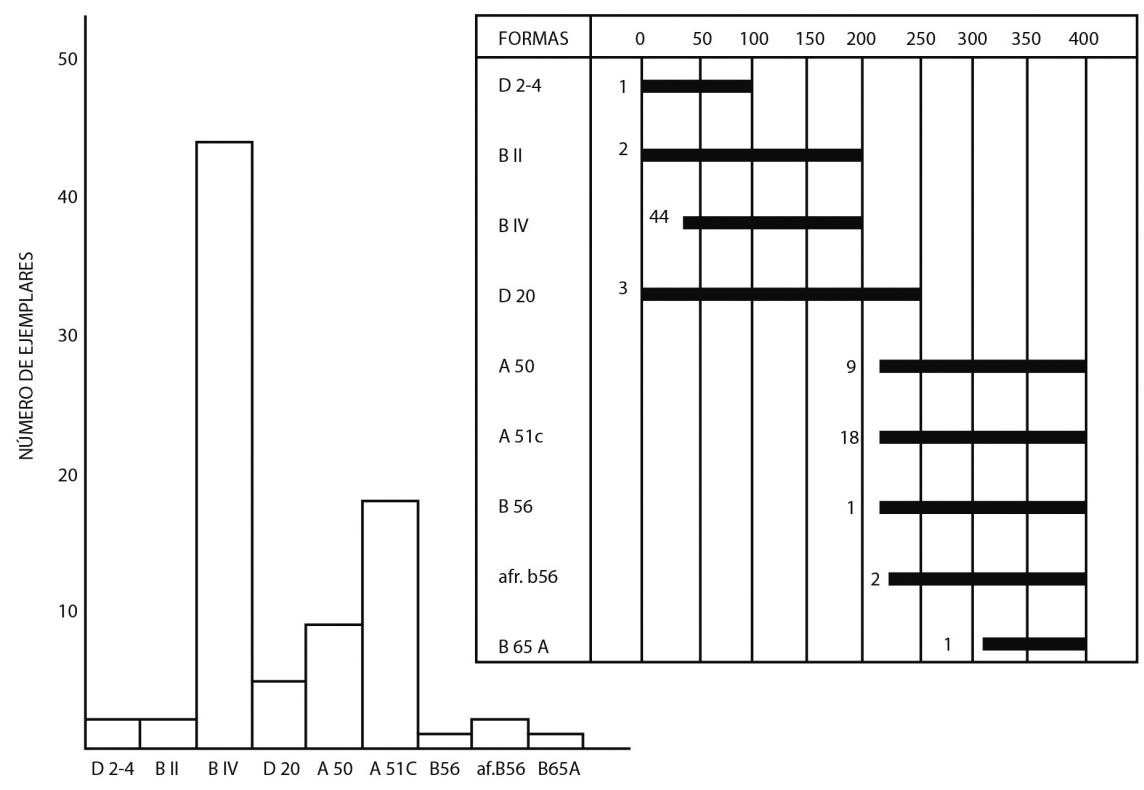

\begin{tabular}{|lllll|}
\hline Cerâmica Incisa & I a & lb & Ila & Ilb \\
a) ungulada duplamente & - & - & - & - \\
b) nervada & - & - & - & - \\
c) "folha-da-acácia" & - & - & + & + \\
d) "folha-de-crucifera" & + & + & + & - \\
e) espiga vertical & - & - & + & + \\
f) "copos"Vila Nova I & - & - & - & + \\
g) caneladuras paralelas & - & - & + & - \\
h) vasos de bojo alto & - & - & + & - \\
i) ziguezagues verticais & - & - & - & + \\
j) ziguezagues horizontais & - & - & + & + \\
k) de "tipo chasseense" & - & - & - & + \\
\hline
\end{tabular}
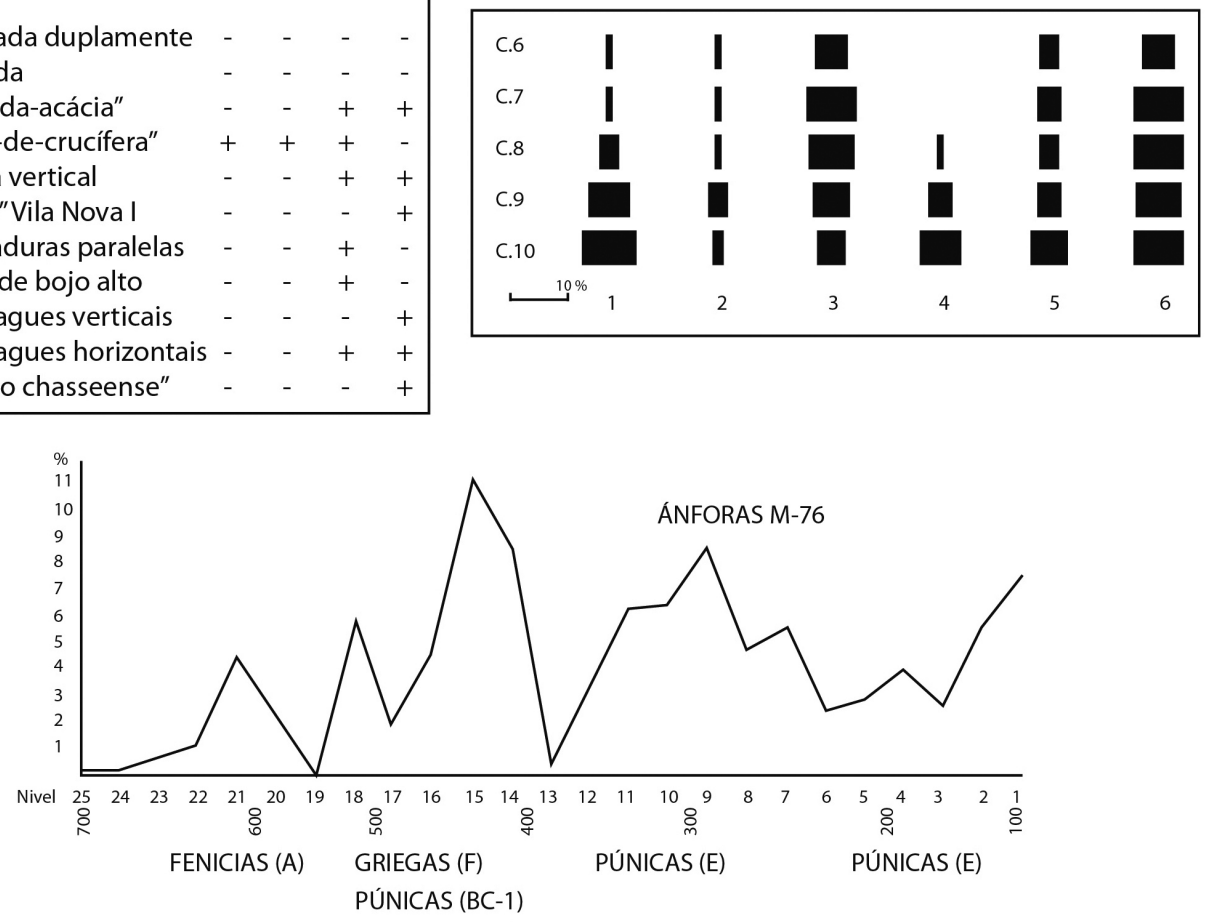

PÚNICAS (BC-2) ITAL (G) ROM $\quad$ R

PÚNICAS (BC-3) CART (I)

Fig. 4. A) Análisis del consumo en la ciudad de Setúbal (Coelho-Soares y Silva, 1978: 173 y figs. 2-3); B) cuantificación en el Castro da Rutura en Portugal (Gonçalves, 1971); C) aplicación conjunta de métodos cuantitativo-estadísticos en Castelo de Alcácer do Sal (Silva et al., 1980-81); d) cuantificación de las ánforas de Cerro Macareno (Pellicer, 1978: fig. 14 vectorizado por los autores a partir de los originales). 
surgieron en la arqueología protohistórica. En 1978 se publican las ánforas de Cerro Macareno, donde se presenta un estudio cronotipológico junto a un esquema evolutivo diacrónico del consumo de las ánforas (Pellicer, 1978: fig. 14) (Fig. 4d).

En 1984 se publican las ánforas romanas de Valencia presentando un primer recuento de fragmentos por tipos, donde se registran de forma individualizada las partes identificadas sintetizando los totales con gráficos de cantidad, aunque sin el uso de estadística (Fernández Izquierdo, 1984: cuadro general).

Ese mismo año aparece el colosal trabajo sobre ánforas tardías de Keay (1984), en el que se aplican por primera vez en territorio peninsular todos los preceptos de la cuantificación desarrollados por la "escuela anglosajona": enumera los principios metodológicos en un apartado propio, expone las características y condicionantes de los distintos tipos de contextos estudiados y desglosa los elementos diagnosticados y cuantificados. Pero sobre todo establece un Número Mínimo de Anforas -Min no Amph- mediante la aplicación ponderada entre el Número Mínimo de Bordes -Min no Rims-, obtenido según el Porcentaje Combinado de Bordes (Comb \% rims) -el principio del EVE - y los fragmentos de fondos (Add feet) y/o asas. Destaca la importancia dada a la necesidad de mostrar de forma "transparente" los datos brutos recopilados, mediante la publicación de todas las tablas de cuantificación que expresan los valores numéricos y porcentuales de los individuos.

Publicado en 1990, destaca el encuentro de Conimbriga dedicado a las ánforas lusitanas donde se incorporan algunas cuantificaciones, como la alfarería del Martinhal (Silva et al., 1990) o el consumo de salazones en São Cucufate de C. Lopes y F. Mayet (1990: 297) basado en fragmentos diagnosticables y sintetizados bajo la forma de una gráfica de barras, y ampliado en una obra monográfica.

Paralelamente observamos el desarrollo de la cuantificación en otros tipos de cerámicas, como es el caso del estudio de Las cerámicas africanas de la ciudad romana de Baetulo (Aquilué, 1987), donde presenta tablas de resultados cuantificadas por clases

(C) Universidad de Salamanca cerámicas, especificando el número y porcentajes de fragmentos con forma o informes por tipos/formas. El estudio de sigillata hispánica en Valencia (Escrivà, 1989) aporta una cuantificación simple por unidades estratigráficas, $\mathrm{y}$, a pesar de carecer de una síntesis global cuantificada, incorpora unas primeras curvas de consumo (Fig 11a); ocasionalmente encontramos cuantificaciones computando los fragmentos de cada forma, calculando porcentajes según zonas de origen de cada producción e incorporando curvas de consumo diacrónico según intervalos regulares establecidos (Montesinos i Martínez, 1991).

Se observa, por tanto, a finales de la década de los 80 la influencia de la arqueología a gran escala, con nuevas aportaciones metodológicas y analíticas en los materiales que surgieron de la mano de los grandes planes de actuación arqueológica, sobre todo en los conjuntos urbanos, como es el paradigmático ejemplo del equipo del TED'A de Tarragona que incorpora sistemas de registro normalizados basados en criterios transversales a la vez que uniformes (TED'A 1989). Los conjuntos cerámicos son contabilizados a través de su agrupación en clases cerámicas, y en función de sus tipos, formas y pastas cerámicas, así como de los fragmentos preservados, contándose los elementos separadamente, y a continuación se calcula el número estimado de piezas -NEP-, aplicándose principios y conceptos paralelos al NMI (Fig. 5B). No obstante, no se hace algún tipo de alusión directa a los protocolos seguidos. En definitiva, durante la década de 1980 en el panorama peninsular los trabajos cuantificados son minoritarios y ofrecen un grado bajo de elaboración, sin integración contextual o estratigráfica, normalmente desprovistos de una lectura porcentual y sin los datos brutos, excepción hecha de algunos estudios como los del TED'a o de S. Keay.

Afortunadamente, en la década posterior la cuantificación cerámica se hará más habitual, especialmente conforme se vayan consolidando las nuevas herramientas digitales capaces de procesar volúmenes cada vez mayores de información. A principios de los 90 encontramos el estudio de las sigillatas de Baelo (Bourgeois y Mayet, 1991), que aporta un cómputo global basado en los fragmentos

Zephyrus, LXXVIII, julio-diciembre 2016, 87-110 


\begin{tabular}{|l|c|c|}
\hline TIPOS & BORDES & PORCENTAJE \\
\hline GRECOITÁLICA & 33 & $8.25 \%$ \\
\hline DRESSEL 1 & 33 & $8.25 \%$ \\
\hline LAMBOGLIA 2 & 4 & $1 \%$ \\
\hline PÚNICAS INDET. & 11 & $2.75 \%$ \\
\hline MAÑ́́ C2-DRESSEL 18 & 6 & $1.5 \%$ \\
\hline PASCUAL 1 & 7 & $1.75 \%$ \\
\hline PÚN-EBUSITANA & 8 & $2 \%$ \\
\hline DRESSEL 2-4 & 141 & $35.25 \%$ \\
\hline DRESSEL 7-11 & 54 & $13.5 \%$ \\
\hline BELTRÁN II & 16 & $4 \%$ \\
\hline HALTERN 70 & 7 & $1.75 \%$ \\
\hline DRESSEL 20 & 9 & $2.25 \%$ \\
\hline DRESSEL 5 & 14 & $3.5 \%$ \\
\hline TRIPOLITANA 1 & 8 & $2 \%$ \\
\hline DRESSEL 30 & 20 & $5 \%$ \\
\hline TARDÍAS & 13 & $3.25 \%$ \\
\hline INDETERMINADAS & 16 & $4 \%$ \\
\hline TOTAL & 400 & $\mathbf{1 0 0 . 0 0} \%$ \\
\hline
\end{tabular}

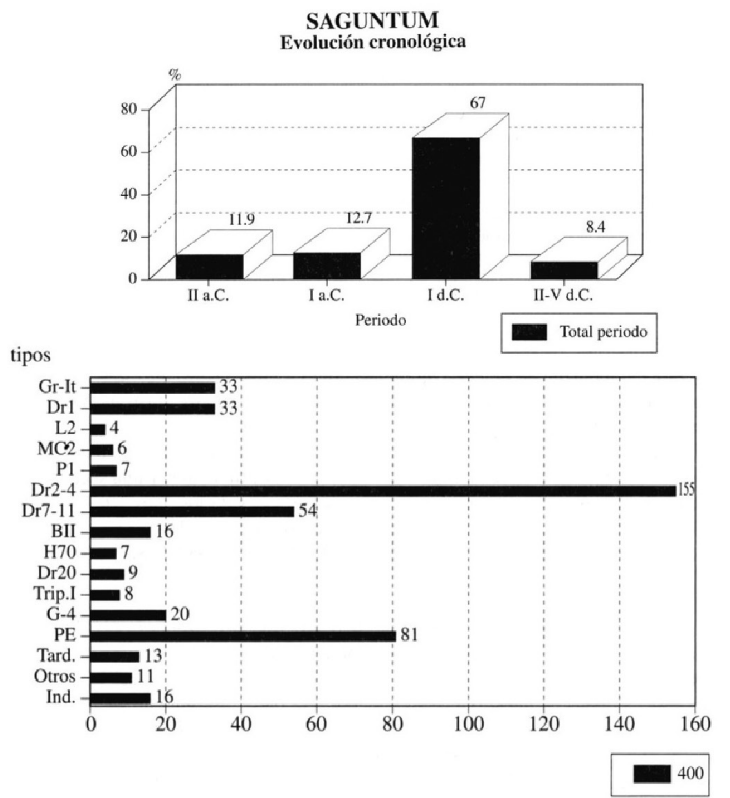

\begin{tabular}{lcccccc}
\hline TIPO & BORDE & BASE & ASA & GALBO & N TOTAL & N.M.I. \\
\hline Grecoitál. & 4 & 1 & - & - & 5 & 4 \\
\hline Dr. 1 & 34 & 15 & 5 & 10 & 64 & 34 \\
\hline Dr. 2/4 & 1 & - & - & - & 1 & 1 \\
\hline Beltrán II & 6 & 1 & - & 2 & 9 & 6 \\
\hline D. 20 & 2 & - & - & - & 2 & 2 \\
\hline Gala 4 & - & - & 1 & - & 1 & 1 \\
\hline Halt. 70 & 1 & - & - & - & 1 & 1 \\
\hline K.I & 2 & 2 & - & - & 4 & 2 \\
\hline K. III & 3 & - & - & - & 3 & 3 \\
\hline K. IV & 1 & - & - & - & 1 & 1 \\
\hline K. VII & 1 & - & - & - & 1 & 1 \\
\hline K. XXV & 3 & 4 & - & - & 7 & 4 \\
\hline K. XXVI & - & 1 & - & - & 1 & 1 \\
\hline K. XXXIV & 1 & - & - & - & 1 & 1 \\
\hline K. LXII & 1 & - & - & - & 1 & 1 \\
\hline Ind. Afric. & 2 & 4 & 1 & 2 & 9 & 4 \\
\hline Indet. & 1 & 5 & - & 7 & 13 & 5 \\
\hline Total & 64 & 33 & 7 & 21 & 125 & 73 \\
\hline
\end{tabular}

(Tabla I). Número total de fragmentos anfóricos y número mínimo de individuos procedentes de Pudrimel.

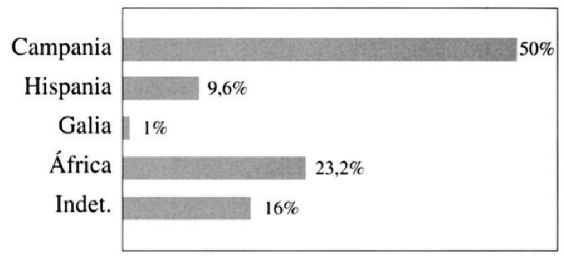

\begin{tabular}{|c|c|c|c|c|c|c|c|c|c|c|c|c|c|c|c|c|c|c|c|}
\hline NIVS. & & 1 & ONEN & & & & & & & & & $\begin{array}{l}\text { ITA } \\
163\end{array}$ & & & & & & $\begin{array}{l}\text { ESTE } \\
13\end{array}$ & iil \\
\hline & T.1 & $\begin{array}{l}\mathrm{Dr} . \\
2 / 4\end{array}$ & $\begin{array}{l}\text { Ob. } \\
74\end{array}$ & P.1 & Dr. & $i ?$ & $\begin{array}{c}\text { Bel. } \\
\text { I }\end{array}$ & $\begin{array}{l}\text { Dr. } \\
12\end{array}$ & $\mathrm{H}_{70}^{\mathrm{Ha}}$ & $\begin{array}{l}\text { Dr } \\
20\end{array}$ & ¿? & $\begin{array}{l}\text { Dr. } \\
1 \mathrm{~A}\end{array}$ & $\begin{array}{l}\text { Dr. } \\
1 \mathrm{~B}\end{array}$ & Ap. & $\begin{array}{c}\mathrm{Dr} \\
{ }_{6}\end{array}$ & $\begin{array}{l}\text { Dr. } \\
2 / 4\end{array}$ & ¿? & Rod. & i? \\
\hline 1.2 & & & & & & & & & & & & 2 & & & & & 4 & & \\
\hline 1.3 & & & & & & & & & & & & 3 & & & & 1 & 11 & & \\
\hline 3 & 1 & & & 1 & & 12 & 3 & & & & 5 & 3 & 2 & & & & 32 & 1 & 18 \\
\hline 5 & & 32 & 8 & 9 & 1 & 335 & 8 & & 2 & 1 & 30 & 1 & & & 1 & 1 & 39 & 1 & 40 \\
\hline 6 & 1 & 12 & 6 & 1 & & 41 & 6 & 1 & 3 & & 15 & 1 & 1 & & & & 5 & & 12 \\
\hline 7.1 & 3 & 28 & 11 & 20 & 1 & 289 & 9 & 1 & 4 & & 40 & 4 & 2 & & & 2 & 12 & 3 & 31 \\
\hline 7.2 & 1 & 7 & 2 & 20 & & 195 & 6 & & & & 14 & & & & & 2 & 19 & 7 & 10 \\
\hline 8 & 1 & 8 & 1 & 1 & & 83 & & & & 1 & 22 & 1 & 1 & 1 & & & 12 & 1 & \\
\hline Tot. & 7 & 87 & 28 & 52 & 2 & 955 & 32 & 2 & 9 & 2 & 126 & 15 & 6 & 1 & 1 & 6 & 134 & 13 & 111 \\
\hline
\end{tabular}

Porcentajes por todos los productos ${ }^{29}$ :

\begin{tabular}{|l|l|l|l|l|l|}
\hline NIVEL /PROD. & TARRACONENSE & BÉTICA & ITALIA & ESTE & TOTAL \\
\hline $\begin{array}{l}\text { Nivel 1 } \\
\text { Vino }\end{array}$ & & & 5 & 5 \\
\hline $\begin{array}{l}\text { Nive13 } \\
\text { Vino } \\
\text { Salazones }\end{array}$ & $2(18,18 \%)$ & $3(27,27 \%)$ & $5(45,45 \%)$ & $1(909 \%)$ & 11 \\
\hline $\begin{array}{l}\text { Nivel 5 } \\
\text { Vino } \\
\text { Aceite } \\
\text { Salazones }\end{array}$ & $50(76,92 \% 0$ & $\begin{array}{l}2(3,07 \%) \\
1(1,53 \%) \\
8(12,30 \%)\end{array}$ & $2(3,07 \%)$ & $1(1,53 \%)$ & 70 \\
\hline $\begin{array}{l}\text { Nivel 6-7 } \\
\text { Vino } \\
\text { Salazones }\end{array}$ & $113(91,90 \%)$ & $\begin{array}{l}7(4,26 \%) \\
23(14,02 \%)\end{array}$ & $16(9,75 \%)$ & $5(3,04 \%)$ & 164 \\
\hline $\begin{array}{l}\text { Nivel } 8 \\
\text { Vino } \\
\text { Aceite }\end{array}$ & $11(73,33 \%)$ & $1(6,66 \%)$ & $\begin{array}{l}3(20 \%) \\
1(6,66 \%)\end{array}$ & & 16 \\
\hline
\end{tabular}

Fig. 5. A) Estudio del caso de ánforas del área de Carthago Nova (Molina, 1997); в) estudio de materiales del pecio de Pudrimel (Pascual, 1998: 266); C) estudio sobre Celsa con estratigrafía y cuantificación (Beltrán Lloris, 1998).

diagnósticos acompañado de histogramas, resultados brutos por categorías, y todo ello convenientemente tabulado y analizado porcentualmente a través del cálculo de hipotéticas medias anuales basadas en los ciclos de producción que abarcan cada uno de los diferentes tipos de sigillata, intentando aproximarse a unos probables valores reales de consumo (Fig. 11c). 
La influencia de la escuela de Lattes y del desarrollo de los protocolos del sysLAT habría a su vez pesado notablemente en los estudios de materiales cerámicos en dos zonas opuestas peninsulares como Granada y Cataluña, puesto que desde finales de los años 80 y sobre todo inicios de los 90 un número cada vez mayor de proyectos de investigación y de intervenciones puntuales aplicaba dichos sistemas, entre los que podemos contar las excavaciones sistemáticas y de urgencia en Granada ciudad o en Guadix, por un lado, y los proyectos como Mas Castellar de Pontos y Puig de Sant Andreu en Gerona o El Vilars d'Arbeca en Lérida, tomando particularmente como base de cuantificación el NMI y el NFR.

Para el ámbito lusitano destaca la sigillata de Represas (Lopes, 1994) para la cual se vuelven a utilizar los cálculos de medias anuales con los años de duración de cada una de las categorías, y añadiendo un análisis comparando fragmentos y número de intervenciones. Por su parte, en el trabajo sobre Cerámica sigillata de Monte Mozinho, T. P. de Carvalho desglosa la información bruta en "Cuadros Descriptivos" y los resultados en histogramas, comparando las importaciones totales con las de otros yacimientos-Baelo, Conimbriga, Numantia y Saguntum- de forma gráfica y porcentual (Carvalho, 1998: figs. LXXIV-LXXVII, n. ${ }^{\text {os } 229-232) . ~}$

Pero es en el estudio de la sigillata de la Alcáçova de Santarém (Viegas, 2003) en el que por primera vez se aplicaron los principios del Protocolo de Beuvray -NFR y NMI-, con pequeñas correcciones para minimizar los efectos de "inflación" inherentes a determinadas formas. Al final, se ofrece toda la información en forma de tablas con los datos brutos y porcentuales. Al tiempo que se utiliza el cálculo de medias anuales para poder hacer comparaciones con otros yacimientos. En varios casos se ha aplicado también la metodología defendida por M. Polak (2000) para la cuantificación de la distribución de frecuencia de los sellos de alfarero. Sin embargo, en algunos de éstos no se explicitan los parámetros cronológicos utilizados en el cálculo de las medias anuales o de frecuencias por categorías y tipos. Pueden destacarse una gran cantidad de estudios en la zona tarraconense oriental, especialmente en relación con Baetulo, Ampurias o la Narbonense, si bien se constata una utilización menor de lo deseable de la cuantificación en los trabajos sobre cerámicas finas, limitada frecuentemente a la confección de simples mapas de difusión y presencia.

Aún menor será el recorrido de la cuantificación aplicado a conjuntos de cerámicas comunes, aunque también a partir de la década de 1990 se empiezan a encontrar trabajos que al componente petrográfico incorporan el cuantitativo. Cabe destacar algunos trabajos reunidos en el volumen Ceràmica comuna romana d'època Alto-Imperial a la Península Ibèrica (Aquilué y Roca, 1995), que incorporan el cálculo de NMI, especialmente los referidos al ámbito tarraconense.

Más esclarecedor es el panorama de los estudios dedicados al mundo de las ánforas. En primer lugar, hemos de señalar el trabajo de J. Molina (1997), que desarrolla un análisis metódico de cuantificación anfórica para tratar de caracterizar el comercio romano y donde analiza prácticamente todos los métodos de cuantificación hasta entonces conocidos para concluir que el método de recuento de bordes presentaba una mayor fiabilidad de resultados en relación al tiempo invertido. Aplicó de forma sistemática dicho método en el estudio de los yacimientos del área de Carthago Nova, presentando todas las tablas de cuantificación y gráficas correspondientes, y apuntando la necesidad de desarrollar correctores estadísticos como el Módulo de Ruptura-MR-.

Igualmente ejemplar es el trabajo que publica sobre Pudrimel, que presenta no sólo las tablas con todos los elementos diagnosticables diferenciados y cuantificados -NFR y NMI-, sino que presenta también el correspondiente apartado sobre la metodología y protocolos empleados (Pascual, 1998: 266). Contemporáneamente aparece el trabajo de Beltrán Lloris (1998) sobre Celsa, en el que se presenta una cuantificación de fragmentos por proveniencia estratigráfica/tipo, y un análisis final del total de fragmentos/tipos cuantificados y de los productos transportados. En la misma línea metodológica se insertan los estudios publicados por Remolà Vallverdú para las ánforas de Tarragona (2000) o de Morais para las de Carvalheiras (1998, 2005), en los 
que se usa el NMI establecido a partir del recuento de bordes.

La utilización progresiva de la cuantificación que se hacía notar en la investigación de la Península Ibérica queda reflejada en el Congreso Internacional Ex Baetica Amphorae de 1998. Una buena parte de los trabajos incluidos en el volumen II presentan ya conjuntos cuantificados, aunque con un predominio de la cartografía de difusión sintética o 'mapas cuantificados' respecto a las tablas detalladas o la publicación detallada de los datos. La proporcionalidad entre los distintos elementos que lo componen -círculos, cuadrados, triángulos, etc.- permite transmitir solo una idea limitada de cuantificación y establecer comparaciones demasiado básicas, aunque permite suplir las lagunas de los meros estudios de presencias (Ramón, 1995; Beltrán Lloris, 2001).

Con la publicación de C. Carreras (2001) del conjunto anfórico del Castro de Campa Torres, se incorpora en la Península Ibérica una nueva metodología de trabajo y una forma diferente de cuantificar que combina el recuento de fragmentos -NFR-, el número de individuos calculado con el EVE y el peso de fragmentos -PFR-, que además se utilizan para generar análisis de densidades. Este método aporta considerables ventajas adicionales a la cuantificación, aunque el elevado grado de especialización que requiere y la falta de hábito en su aplicación han provocado su menor impacto en el ámbito peninsular.

Sin embargo, es cierto que en la última década se constata un progresivo predominio de los métodos cuantitativos 'sencillos': NMI a partir del recuento directo de bordes o del número de individuos ponderado, de acuerdo con el Protocole de Beuvray. Para Hispalis destaca el trabajo de E. García Vargas (2007), que cuantifica NMI a partir del EVE de bordes sin presentar los datos brutos. En este trabajo destaca el apartado de las lecturas analíticas: extrapolación del cálculo de una media del consumo anual y cálculo de las importaciones en litros de producto contenido (García Vargas, 2007: 334-335), todo ello acompañado de los respectivos histogramas de síntesis. Podemos seguir con la sigillata de Mirobriga (Quaresma, 2012), las ánforas

(C) Universidad de Salamanca de Vigo (Fernández Fernández, 2014) o Aeminium (Silva et al., 2015), todos ellos siguiendo metodologías de cuantificación clara y presentando abundante y detallada documentación sintetizada en cuadros o tablas.

En general, en la actualidad observamos una mayor presencia y conocimiento de métodos cuantitativos, aunque faltan protocolos de registro que permitan el acceso a los valores brutos/originales y demás información cuantificable. En este sentido cabe destacar el trabajo de las ánforas romanas de Lugo (Carreras y Morais, 2011), en el que, a pesar de haberse utilizado un método menos frecuente, como es el PFR, junto con NFr, EVE y cálculo de densidades, la presentación de todos los datos brutos permite establecer equivalencias en algunos niveles de información comunes a los obtenidos con otros métodos. De ahí que pensemos que el problema no está tanto en el método de cuantificación utilizado, dependiente de la naturaleza del conjunto estudiado y de los objetivos de la investigación, sino en el registro y presentación de los datos de forma sistematizada.

Para terminar, nos gustaría hacer referencia y llamar la atención de forma particular al desarrollo de sistemas de registro preparados ya para asumir los principales sistemas de cuantificación a partir de la automatización de los protocolos. Es el caso de SIRA -Sistema Informatizado de Registro Arqueológico- desarrollado en la Universidad de Granada. Se trata de una herramienta de gestión del registro arqueológico que garantiza una sistematización de los protocolos de cuantificación a la par que posibilita su integración con los resultados de las excavaciones arqueológicas, pudiendo generar autoinformes de diversos tipos (Adroher et al., 2015: 47).

\section{Propuesta de protocolos de registro y análisis de conjuntos cerámicos}

La enorme heterogeneidad de métodos y la desigual forma de acometer el registro cuantitativo han complicado el desarrollo de los análisis de este tipo. Sin un sistema de registro normalizado se hace 
100 A. M. Adroher, C. Carreras, R. de Almeida, A. Fernández, J. Molina y C. Viegas / Registro para la cuantificación...

\begin{tabular}{|c|c|c|c|c|c|c|c|c|c|}
\hline \multicolumn{10}{|c|}{ Sigillées } \\
\hline \multirow[b]{2}{*}{ Formes } & \multicolumn{3}{|c|}{ [488] } & \multicolumn{3}{|c|}{ [519] } & \multicolumn{3}{|c|}{ Total } \\
\hline & $\begin{array}{l}\text { № } \\
\text { fgs }\end{array}$ & NMI & $\%$ NMI & $\begin{array}{l}\text { № } \\
\text { fgs }\end{array}$ & NMI & $\%$ NMI & $\begin{array}{l}\text { № } \\
\text { fgs }\end{array}$ & NMI & $\%$ NMI \\
\hline Drag. 27 (TSS) & 0 & 0 & $0,0 \%$ & 1 & 1 & $3,7 \%$ & 1 & 1 & $0,8 \%$ \\
\hline Drag. 27 (TSH) & 1 & 1 & $1,0 \%$ & 0 & 0 & $0,0 \%$ & 1 & 1 & $0,8 \%$ \\
\hline Hayes 50 (TSA C) & 1 & 1 & $1,0 \%$ & 1 & 1 & $3,7 \%$ & 2 & 2 & $1,6 \%$ \\
\hline Hayes 58 (TSA C) & 2 & 2 & $2,0 \%$ & 0 & 0 & $0,0 \%$ & 2 & 2 & $1,6 \%$ \\
\hline Bols à listel (TSA C) & 1 & 1 & $1,0 \%$ & 0 & 0 & $0,0 \%$ & 1 & 1 & $0,8 \%$ \\
\hline Hayes 52 (TSA D) & 1 & 1 & $1,0 \%$ & 0 & 0 & $0,0 \%$ & 1 & 1 & $0,8 \%$ \\
\hline Hayes 59 (TSA D) & 15 & 10 & $10,2 \%$ & 9 & 6 & $22,2 \%$ & 24 & 16 & $12,8 \%$ \\
\hline Hayes 60 (TSA D) & 2 & 1 & $1,0 \%$ & 0 & 0 & $0,0 \%$ & 2 & 1 & $0,8 \%$ \\
\hline Hayes 61 (TSA D) & 23 & 23 & $23,5 \%$ & 6 & 6 & $22,2 \%$ & 29 & 29 & $23,2 \%$ \\
\hline Hayes 62/EI Mahrine 5 (TSA D) & 5 & 5 & $5,1 \%$ & 1 & 1 & $3,7 \%$ & 6 & 6 & $4,8 \%$ \\
\hline Hayes $62 / 64$, variante Fulford 6 (TSA D) & 1 & 1 & $1,0 \%$ & 0 & 0 & $0,0 \%$ & 1 & 1 & $0,8 \%$ \\
\hline Hayes 63 (TSA D) & 3 & 3 & $3,1 \%$ & 1 & 1 & $3,7 \%$ & 4 & 4 & $3,2 \%$ \\
\hline Hayes 67 (TSA D) & 13 & 10 & $10,2 \%$ & 4 & 4 & $14,8 \%$ & 17 & 14 & $11,2 \%$ \\
\hline El Mahrine 14 (TSA D) & 26 & 23 & $23,5 \%$ & 3 & 3 & $11,1 \%$ & 29 & 26 & $20,8 \%$ \\
\hline Hayes 71 (TSA D) & 1 & 1 & $1,0 \%$ & 0 & 0 & $0,0 \%$ & 1 & 1 & $0,8 \%$ \\
\hline Hayes 73 (TSA D) & 1 & 1 & $1,0 \%$ & 0 & 0 & $0,0 \%$ & 1 & 1 & $0,8 \%$ \\
\hline Hayes 76 (TSA D) & 1 & 1 & $1,0 \%$ & 0 & 0 & $0,0 \%$ & 1 & 1 & $0,8 \%$ \\
\hline Hayes 80A-B/81B (TSA D) & 10 & 10 & $10,2 \%$ & 3 & 2 & $7,4 \%$ & 13 & 12 & $9,6 \%$ \\
\hline Hayes 91 (TSA D) & 20 & 3 & $3,1 \%$ & 6 & 2 & $7,4 \%$ & 26 & 5 & $4,0 \%$ \\
\hline Total & 127 & 98 & $100 \%$ & 35 & 27 & $100 \%$ & 162 & 125 & $100 \%$ \\
\hline
\end{tabular}

\begin{tabular}{|c|c|c|c|c|c|c|c|c|}
\hline Conteúdo & Origem & Tipo & $\begin{array}{l}\text { Total } \\
\text { NMI }\end{array}$ & $\%$ & $\begin{array}{c}\text { Total } \\
\text { NMI } \\
\text { por } \\
\text { origem }\end{array}$ & $\%$ & $\begin{array}{c}\text { Total } \\
\text { NMI } \\
\text { por } \\
\text { produto }\end{array}$ & $\%$ \\
\hline Vinho & Itália & $\begin{array}{l}\text { Greco-itálicas } \\
\text { Dressel } 1 \\
\text { Lamboglią } 2\end{array}$ & $\begin{array}{c}2 \\
38 \\
5\end{array}$ & $\begin{array}{c}0.7 \\
13.6 \\
1.8\end{array}$ & 45 & 16.1 & 45 & 16.1 \\
\hline Peixe & Tripolitania & Mañá C2a & 5 & 1.8 & 5 & 1.8 & 5 & 1.8 \\
\hline Peixe & U/terior & $\begin{array}{l}\text { Castro Marim } 1 \\
\text { T.9.1.1.1 } \\
\text { Mañá Pascual A4 } \\
\text { Mañá C2b } \\
\text { Classe } 67 \\
\text { Dressel } 12\end{array}$ & $\begin{array}{c}99 \\
4 \\
4 \\
78 \\
24 \\
2\end{array}$ & $\begin{array}{c}35.5 \\
1.4 \\
1.4 \\
28 \\
8.6 \\
0.7\end{array}$ & 211 & 75.6 & 211 & 75.6 \\
\hline Indeterm. & Ulterior & $\begin{array}{l}\text { Greco-itálica } \\
\text { Dressel } 1 \\
\text { Forma D evoluída }\end{array}$ & $\begin{array}{l}2 \\
6 \\
5\end{array}$ & $\begin{array}{l}0.7 \\
2.2 \\
1.8\end{array}$ & 13 & 4.7 & 13 & 4.7 \\
\hline Vinho/Defrutum & Ulterior & Haltern 70 & 5 & 18 & 5 & 18 & 5 & 18 \\
\hline Total & & & 279 & 100 & 279 & 100 & 279 & 100 \\
\hline
\end{tabular}

\begin{tabular}{|c|c|c|c|c|c|c|c|c|}
\hline Conteúdo & Origem & Tipo & $\begin{array}{l}\text { Total } \\
\text { NMI }\end{array}$ & $\%$ & $\begin{array}{c}\text { Total } \\
\text { MNI } \\
\text { por } \\
\text { origem }\end{array}$ & $\%$ & $\begin{array}{c}\text { Total } \\
\text { NMI } \\
\text { por } \\
\text { produto }\end{array}$ & $\%$ \\
\hline Peixe & Bética & $\begin{array}{l}\text { Dressel 7/11 } \\
\text { Dressel } 14 \\
\text { Beltrán II A } \\
\text { Beltrán II B }\end{array}$ & $\begin{array}{c}25 \\
6 \\
2 \\
17\end{array}$ & $\begin{array}{c}21.2 \\
5.1 \\
1.7 \\
14.4\end{array}$ & & & 50 & 42.4 \\
\hline Defrutum & & Haltern 70 & 41 & 34.8 & & & 41 & 34.8 \\
\hline Azeite & & $\begin{array}{l}\text { Haltern } 71 \\
\text { Dressel } 20\end{array}$ & $\begin{array}{c}1 \\
23\end{array}$ & $\begin{array}{c}0.8 \\
19.5\end{array}$ & 115 & 97.5 & 24 & 20.3 \\
\hline Vinho & Gália & Gauloise 4 & 1 & 0.8 & 1 & 0.8 & 1 & 0.8 \\
\hline Indet. & N. África & Tripolitana II & 2 & 1.7 & 2 & 1.7 & 2 & 1.7 \\
\hline \multicolumn{3}{|l|}{ Total } & 118 & 100 & 118 & 100 & 118 & 100 \\
\hline
\end{tabular}

\begin{tabular}{|c|c|c|c|c|c|c|c|c|c|c|c|}
\hline Clase & \begin{tabular}{|l|} 
Producción \\
\end{tabular} & Forma & \begin{tabular}{|l|} 
Observaciones \\
\end{tabular} & \begin{tabular}{l|l} 
TF & \\
\end{tabular} & C & B & $\mathbf{F}$ & A & $\mathbf{P}$ & NMI & Fig. 169-174 \\
\hline \multirow{10}{*}{ Vajilla Fina } & TSBT roja & Forma 7 & & 10 & 3 & 7 & & & & 10 & $1-7$ \\
\hline & & Forma 8 & & 13 & 2 & 11 & & & & 13 & $1-13$ \\
\hline & & Forma 12 & & 11 & 1 & 10 & & & & 11 & $1-11$ \\
\hline & & Forma 13 & & 1 & & 1 & & & & 1 & 1 \\
\hline & & Forma 17 & & 1 & & 1 & & & & 1 & 1 \\
\hline & & Forma 18 & & 4 & & 4 & & & & 4 & $1-4$ \\
\hline & & Forma 3 & & 1 & & 1 & & & & 1 & 1 \\
\hline & & Forma 11 & & 1 & & 1 & & & & 1 & 1 \\
\hline & & Forma 21 & & 3 & 1 & 2 & & & & 3 & $1-3$ \\
\hline & & Indeterminadas & & 114 & & & 89 & & 25 & & $1-24$ \\
\hline \multicolumn{4}{|l|}{ Total/F } & 159 & 7 & 38 & 89 & & 25 & 45 & \\
\hline
\end{tabular}

Fig. 6. A) Basurero en la factoría de Tróia (Pinto et al., 2012); B) análisis de los conjuntos de ánforas de las principales ciudades romanas del actual Algarve (Viegas, 2011); C) Vigo, cuantificación de sigillatas (Fernández Fernández, 2014). 
difícil contrastar los datos, comparar las magnitudes de distintos yacimientos o analizar proporcionalmente los mecanismos de producción, distribución y consumo. Por ello resulta absolutamente necesario determinar unos protocolos de registro para la cuantificación que permitan contrastar las investigaciones, establecer pautas y favorecer la comunicabilidad de los resultados. La experiencia nos ha enseñado que el establecimiento de protocolos científicos favorece el crecimiento de la información primaria al conjunto de la comunidad científica, multiplicando las posibilidades de desarrollar investigaciones derivadas.

Las cuantificaciones estandarizadas, como la que se propone, permitirán integrar conjuntos cerámicos de un mismo yacimiento, pero de distintas excavaciones, incluso realizadas en distintos periodos. Permitirá observar tendencias a nivel espacial, intrasite, que ayudarán a reconocer distintas funciones, desarrollos urbanísticos y tendencias económicas de las cuales, hoy en día, apenas tenemos intuiciones. Pero, sobre todo, la cuantificación nos permite analizar distribuciones de productos concretos a gran escala, intersite, como el caso de las sigillatas o ánforas que cubren amplios territorios del Imperio Romano.

Sobre la necesidad de cuantificar no podemos entrar de forma profunda, pero no cabe duda de que la arqueología cuantitativa demostró la eficacia de establecer ciertos protocolos en la recogida de datos y en la elaboración de analísticas subsecuentes; de hecho, el importante desarrollo de la arqueometría en los últimos dos decenios es una consecuencia directa de los cambios producidos en la valoración técnica que los equipos de investigación dan a la estadística en particular y a las matemáticas en general; incluso muchas de las técnicas aplicadas desde otros ámbitos usan estos métodos para alcanzar modelizaciones que permitan un mejor conocimiento del objeto de estudio de cada disciplina, además de acelerar la velocidad de transmisión de los datos entre investigadores, facilitando el acceso a las muestras analizadas según el interés de cada línea de trabajo.

En el caso de las ciencias sociales en general, de la Historia como tal y de la arqueología en particular, no cabe duda, como se indicaba al principio, de que los grandes modelos interpretativos sobre el

(C) Universidad de Salamanca comportamiento del comercio, de las costumbres culturales, de las evoluciones tecnológicas tienen como punto de partida el uso de métodos estadísticos que permitan observar el alcance de dichos cambios. Pero no solamente desde un punto de vista meramente interpretativo, sino en el mismo proceso de valoración y analítica de los datos podemos ver un importante avance gracias al uso de métodos de cuantificación, como la identificación etnocultural de un grupo social determinado en un espacio y en un tiempo concretos, la adecuación de cronologías de materiales estratificados en un yacimiento mediante el comportamiento porcentual de cada una de las series en él atestiguadas, establecimiento del nivel de homogeneidad de un estrato por la representación de las intrusiones que contiene, que son algunas de las ventajas que presenta el uso de sistematización de datos mediante protocolos de cuantificación. La repetición del uso de sistemas predefinidos permite, efectivamente, dibujar líneas de comportamiento del material que apoyan los procesos analíticos e interpretativos.

Y por este motivo, ante el importante incremento de actividades arqueológicas, y comprobando que cada vez en menos ocasiones se realizan estudios adecuados y sobre todo útiles para contar con un mínimo de parámetros que permitan establecer esas comparativas y desarrollar teorías de alcance medio -base de nuestra disciplina-, hemos unido la experiencia de un conjunto de investigadores que trabajan en ámbitos sobre protohistoria y época romana, en espacios tan amplios que acogen todo el Mediterráneo Occidental y la Europa Occidental, lo que ha permitido plantear un modelo que compagine las largas experiencias acumuladas por los propios autores, así como por la amplia bibliografía existente respecto a este tema.

Lógicamente eso significa que el protocolo, como tal, y al completo, aún no ha sido puesto en práctica de forma global, pero, ya que se compone de un agregado de múltiples modelos cuantitativos, podemos decir que todos ellos han sido ensayados en diversos ámbitos, como demuestra largamente el prefacio a esta propuesta que engalana el cuerpo inicial de este trabajo. 

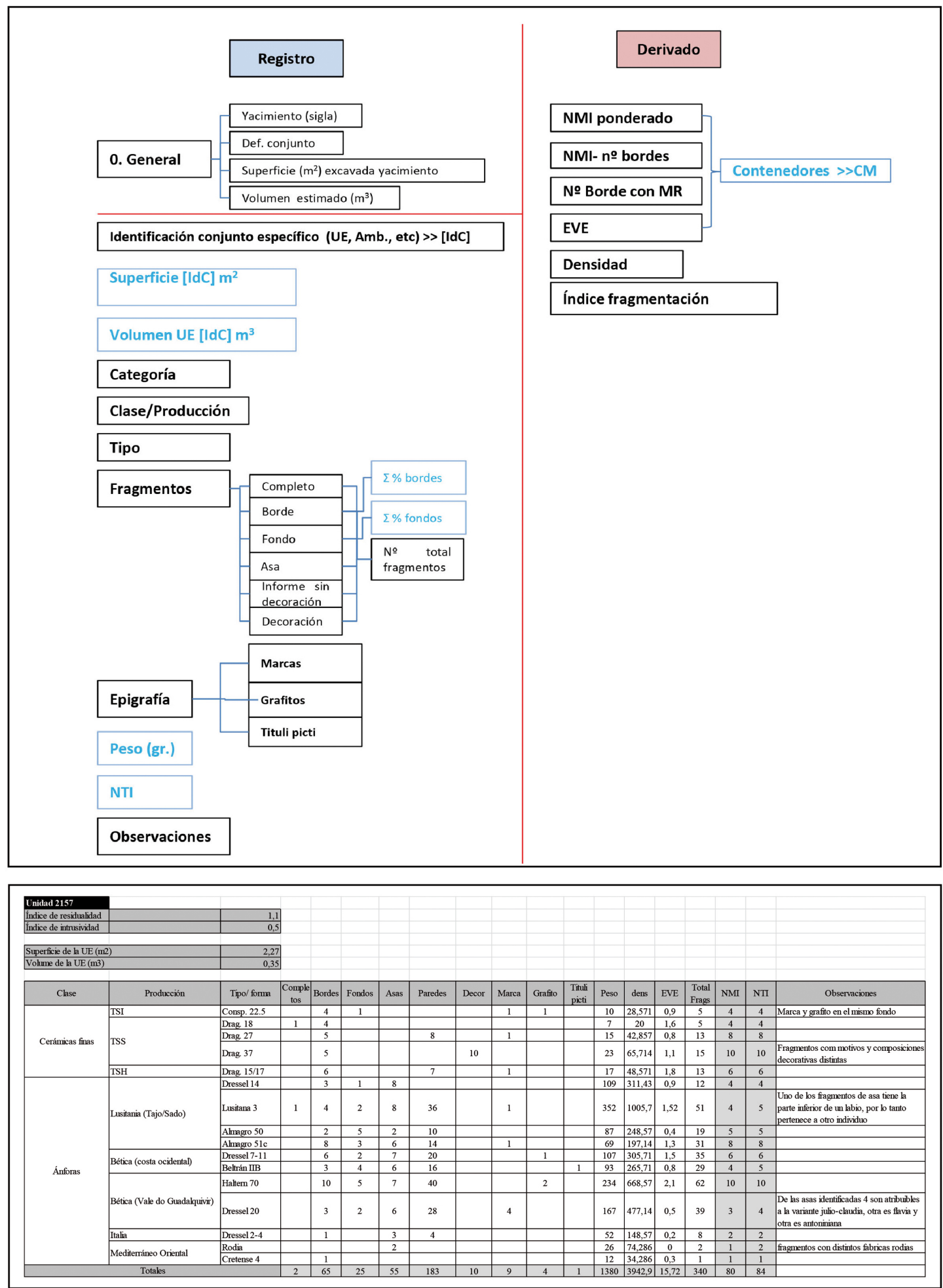

Fig. 7. Propuestas para formulación, ordenación, presentación y publicación del Protocolo de Cuantificación de Cerámicas Arqueológicas de Sevilla (PRCS/14): A) estructura básica del registro; B) desarrollo del modelo de ficha con tabla Excel. 
Se plantea, por tanto, un protocolo de registro con una serie de campos -obligatorios y opcionales- que habrían de ser contemplados a la hora de desarrollar de forma particular, en función de las necesidades de cada investigador o yacimiento, las hojas de cálculo o bases de datos con las que implementar el registro material cuantificable. Un protocolo útil debería incorporar una serie de datos mínimos de modo que pudieran ser visualizados rápidamente y comprendidos de forma automática. No obstante, hay que buscar un equilibrio en los datos que se aporten, de modo que la mayor parte de arqueólogos sea capaz de completar el registro, mientras que quien estime poder llegar más lejos pueda hacerlo completando la totalidad de los datos que se proponen en una ficha ideal. De ahí que se propongan unos campos obligatorios junto a otros opcionales, que aumentarían la calidad y la posibilidad de contrastar los resultados.

Asimismo distinguiremos los registros directos y los registros derivados. Registro directo es aquella parte de la ficha que debe ser completada por el usuario, mientras que por registro derivado entendemos campos cuyos datos se generan automáticamente a partir de los registros directos, al derivarse de éstos. Por otra parte, los campos obligatorios serán marcados con cursivas, mientras que los voluntarios irán sin ninguna especificación básica (Fig. 8).

\subsection{En registro directo}

\section{Yacimiento}

Indica el complejo o sitio arqueológico en el cual se ha practicado la intervención arqueológica específica que se está analizando.

\section{Excavación}

Se trata de la intervención sobre la que se está trabajando en sentido estricto, que incorpora el año de dicha intervención, así como los detalles de la/s campańa/s, la referencia al área de excavación, sector o cualquier otro tipo de referencia de localización.

(C) Universidad de Salamanca

\section{Definición de conjunto}

El conjunto estudiado puede formar parte de un complejo estructural que define la entidad funcional del espacio en su relación con el material, como centro productivo o receptor, sea distributivo primario, secundario o simplemente consumidor del bien. Igualmente, hay que tener en cuenta si la matriz sedimentaria donde se encuentra el material objeto del estudio está en posición primaria o secundaria respecto al complejo estructural funcional.

\section{Superficie excavada}

Indica en metros cuadrados el área total excavada del yacimiento arqueológico del que procede el conjunto cerámico estudiado. Esta variable permitirá estimar la proporción que el conjunto de material representa respecto al yacimiento en sí, poniendo en relación cantidad de material y superficie excavada, con el fin de mejorar las posibilidades de comparación externa, como una muestra de un universo definible.

\section{Volumen estimado}

Se trata de una medida similar a la anterior, pero incidiendo en el volumen en metros cúbicos de tierra excavada, si es que se cuenta con los suficientes datos para poder calcularlo.

\section{Identificación del conjunto especifico}

Es necesario contextualizar adecuadamente el material objeto de estudio, por lo que de alguna manera se deberá introducir la información de la procedencia específica; preferentemente nos referimos a unidades de recogida, como cuadrícula o unidad estratigráfica (UE). Pero tampoco es desdeñable una información de contextualización funcional, como el ambiente, el departamento o el complejo estructural con el cual se asocia el conjunto estudiado.

Superficie del conjunto específico

Se indicará la superficie excavada a la que se asocia el conjunto específico al que se refiere el apartado anterior en metros cuadrados. Se trata de un campo opcional puesto que es muy posible que no se disponga de esta información detallada o específica, 
sobre todo en excavaciones antiguas o materiales depositados en almacenes y museos. Este campo, junto con el de volumen, aun siendo opcional resulta muy interesante ya que permite desarrollar análisis proporcionales de los conjuntos cerámicos, accediendo de esta forma a realizar comparaciones de densidad entre distintos conjuntos específicos, contextos o entre diferentes yacimientos, estableciendo la verdadera importancia en la relación de presencia de un material dado en un espacio o en otro.

Volumen del conjunto específico

Como en el campo anterior, si existe esa posibilidad, se indicará el volumen del conjunto específico en metros cúbicos. Es una medida mucho más descriptiva y completa a la hora de contextualizar la representatividad de la presencia/ausencia de un material dado, aunque hay que reconocer que es mucho más compleja de determinar.

\section{Categoría cerámica}

Se entiende por categoría los grandes grupos de clases asociados por similitudes funcionales-tecnológicas básicas: vajilla de mesa (fina y común), cerámica de cocina y contenedores de transporte/ almacenaje.

\section{Claselproducción cerámica}

Por clase o producción cerámica entendemos el taller o conjunto de talleres regionalmente asociados entre sí que producen unas formas semejantes con características tecnológicas similares (campaniense $\mathrm{A}$ o ánforas apulas). Se tenderá a establecer generalidades que permitan agrupar varios talleres o centros de producción en una sola clase cerámica, necesariamente a partir del análisis y caracterización de pastas cerámicas, barnices, engobes y demás elementos ceramológicos.

Tipo cerámico

Sin entrar en disquisiciones sobre el valor cultural o social del concepto de tipo, nos limitaremos a decir que se trata de un conjunto de valores morfológicos y morfométricos que tradicionalmente han sido utilizados por los investigadores para distinguir

(C) Universidad de Salamanca dos piezas diferentes procedentes de un mismo taller o centro de producción. Los tipos vienen normalmente predeterminados en cada clase cerámica por los estudios que se hayan realizado en torno a ella. Se debería explicitar claramente la tipología utilizada, ya que no es lo mismo decir en Campaniense A Lamboglia 23 que Morel F1122a, ya que podrían tener propuestas cronológicas distintas.

\section{Fragmentos}

Se indicará el número de fragmentos de cada parte cerámica y por tipo -bordes, asas, fondos, cuellos, informes, etc.-. En ningún caso un fragmento se deberá incluir en dos campos. En caso de tener varios elementos se privilegia de la siguiente forma: primero el borde, después el fondo, el asa y, finalmente, el resto -decoración y amorfos-. Complejos son los casos de las lucernas, donde proponemos que los orificios de mecha en la piquera sean considerados como bordes, siendo el resto paredes informes, incluidas todas las zonas del disco. Solo en los casos de lucernas abiertas como las de tradición fenopúnica y algunas de las griegas clásicas se podrían seguir utilizando los criterios de la cerámica vascular. Otro problema son las piqueras o vertederas de piezas concretas como los denominados 'vasos cerveceros' ibéricos o los de los morteros. En el primer caso podrían entrar en el ámbito de las decoraciones, mientras que los segundos, por su ubicación en el perfil de la pieza, deben ser considerados bordes.

Equivalencia de bordes (Eve de borde)

El equivalente de vasija -EvE- (Orton et al., 1993: 172) define cada fragmento como una porción de la vasija completa, aunque por motivos prácticos se suele limitar a contabilizar la proporción en bases y bordes -sumando ambos resultados y dividiéndolo por dos- o con frecuencia se reduce al denominado equivalente de borde -EVE de borde-. Este valor se calcula a partir de la suma de los porcentajes conservados de cada fragmento de borde -o fondo, en su caso- respecto al diámetro completo. El resultado final es fácilmente comprensible, ya que o bien se comparan números totales o se divide por $100 \mathrm{el}$ sumatorio para obtener el número de vasos a partir 
de los bordes. Los cálculos se realizan sencillamente con un medidor de diámetro que permita el cálculo de segmentos de arcos. Adicionalmente y de forma opcional de puede incorporar la Equivalencia de fondos -EVE de fondos-, calculada de la misma manera que el anterior pero con los fondos.

\section{Epigrafia}

Se registrará de forma sistemática la cantidad de fragmentos que contengan marcas/sellos -anteriores a la cocción-, tituli picti o grafitos - posteriores a la cocción- del conjunto estudiado. De esta forma se podrán cuantificar y establecer proporciones de marcado exactas para los distintos tipos cerámicos y sus diferentes producciones. Algo especialmente interesante para categorías como las ánforas o familias como las sigillatas. Se debe incluir tan sólo el número de fragmentos marcados, ninguna otra referencia.

\section{Peso en gramos}

Se trata del peso en gramos de todo el conjunto tipológico que se esté estudiando. Este valor es interesante pues puede informar acerca de la entidad del conjunto así como de la posibilidad de desplazamiento respecto a su posición primaria, pues la erosión provoca pérdida de materia de los fragmentos. Cuando exista un registro suficiente podrá calcularse un equivalente de peso medio de fragmento $y$, consecuentemente, una tabla de conversión de peso de fragmento medio para cada tipología.

\section{Número Tipológico de Individuos o NTI}

Este campo incorpora aspectos analíticos no contemplados en los otros y trata de considerar cualquier factor que permita identificar los diferentes vasos presentes en el conjunto, bajo cualquier premisa -estado de conservación, decoraciones, elementos amorfos clasificables, etc.-. En todo caso el usuario tendrá la opción de rellenar este campo manualmente, si bien habrá que explicar los criterios utilizados para establecer el valor de esta variable.

\section{Observaciones}

Aquí deberá introducirse cualquier apreciación que complete la información recogida en esta ficha, y específicamente los criterios utilizados para el establecimiento del NTI.

\subsection{Registros derivados}

Se trata de campos cuyos datos se generan automáticamente, a partir de los registros previos aportados en los campos anteriores y en función de su grado de detalles.

\section{Número Total de Fragmentos (NFR)}

Es el sumatorio de perfiles completos, bordes, fondos, asas, decoraciones y amorfos sin decorar del campo fragmentos del registro. Suma todos los fragmentos sin ningún otro tipo de validación matemática.

\section{Número Minimo de Individuos (NMI)}

Se establece eligiendo la cantidad mayor del número de bordes, asas y el de fondos, considerando de esta forma todos los fragmentos diagnóstico de un tipo concreto, después de agrupar y asignar los fragmentos que no encajan pertenecientes al mismo individuo/tipo. En caso de que no exista ningún fragmento de borde o fondo de un tipo el valor será 1 , si se han detectado fragmentos informes de ese tipo cerámico. También se puede realizar solo el NMI de bordes, en el que la aproximación se realiza a partir de la cuantificación del número de fragmentos de bordes.

\section{Estimación del Equivalente de Vasija (EVE)}

Esta variable desarrollada por los estudios estadísticos de Orton (1993: 172) se calcula con la media de la suma de los valores de equivalencia de bordes y equivalencia de fondos de los campos de registro. Se trata de una estimación aproximada de vasos de un conjunto.

\section{Número de bordes corregido por su Módulo de Ruptura (MR)}

La aplicación del coeficiente Mr permite desarrollar recuentos directos de bordes corrigiendo el distinto grado de fragmentación o ruptura de cada 
tipo cerámico, especialmente de sus bordes (Mateo y Molina, 2016). El módulo de ruptura de un determinado tipo se obtiene mediante el promedio de los diferentes porcentajes de borde conservados. Para los contenedores anfóricos se han confeccionado unas tablas que van incorporando nueva información en nuevas versiones (Mateo y Molina, 2016).

\section{Cálculo de volumen contenido corregido con Índices de Capacidad Media (CM)}

En el caso de las cerámicas contenedoras, especialmente para las de transporte de mercancías, los análisis cuantitativos pueden perfeccionarse analizando las proporciones del contenido que llegan a un determinado yacimiento, y no tanto el contenedor en sí. Por ello, y ante la enorme variabilidad de capacidades de los distintos contenedores, especialmente entre las ánforas, se están desarrollando índices correctores medios de capacidad - CM- para cada tipo que representarán su capacidad media, con el fin de cuantificar no los envases, sino sus contenidos.

\section{Densidad}

Este valor muestra la representatividad del tipo cerámico en estudio en relación al contexto donde aparece, puesto que no podemos equiparar la presencia de un número determinado de vasos en un gran contexto estratigráfico, como un simple relleno, con una alta concentración de ese material en un espacio reducido como sucedería en un depósito. Igualmente, en dos yacimientos contemporáneos ubicados en posiciones distintas en una red comercial -un puerto de costa y un pequeńo asentamiento rural de montaña- la cantidad esperada de material comercial es necesariamente distinta, $y$ si no tenemos en cuenta la densidad, un valor artificial como la cantidad de excavaciones realizadas en ambos yacimientos podría dibujar una situación inversa. Es decir, que hubiese más material en una zona de consumo interno fuera de las principales vías de comunicación que en un centro de consumo y redistribución primario. Por tanto, la densidad es un índice corrector respecto a la cantidad de intervenciones arqueológicas realizadas y la extensión de las mismas sobre distintos yacimientos y contextualiza adecuadamente las circunstancias del hallazgo.

\section{Recomendaciones finales}

El uso de varios sistemas diferentes de cuantificación sobre el mismo conjunto ha sido testeado sobre algunos contextos privilegiados, a modo de muestreo, como es el caso del depósito votivo del s. IV a. C. en la calle Zacatín, en Granada (Adroher et al., 2015), lo que no hace sino valorar la necesidad de que cada autor considere diversas opciones a la hora de aproximarse al registro arqueológico y tratar de analizarlo de forma adecuada.

Por otra parte, a modo de conclusiones entendemos que se pueden realizar una serie de recomendaciones tanto a los investigadores como a quienes valoren tanto los proyectos de investigación -entidades académicas o privadas- como sus resultados -revistas y editoriales en general-, acerca de ciertos requerimientos en los que insistir a la hora de considerar como valor de calidad en el momento de evaluar ciertos ítems.

- Insistimos en que lo que se cuantifica es lo que hay, y no lo que hubo. En ciertas condiciones podemos aproximarnos a lo que existió en el pasado, pero la casi totalidad de las ocasiones solo tenemos la oportunidad de saber lo que tenemos entre manos.

- Es necesario contrastar información, por lo que debe en todos los casos recurrirse a sistemas de cuantificación normalizados; hasta tal punto es así que las revistas científicas con control de calidad deberían tener en cuenta la utilización de estos sistemas para aceptar publicaciones relacionadas con análisis cerámicos contextualizados, no así, obviamente, con casos particulares o materiales fuera de contexto.

- Debemos tender a publicar la totalidad del material, y no una selección de un contexto, y eso se refiere tanto a las tablas como a las imágenes, pues en ocasiones piezas que pueden pasar inadvertidas a un investigador pueden ser reconocidas 
por otro. Eso significa que no puede realizarse ninguna selección de la muestra, lo que resultaría del todo injustificable. No obstante, también es cierto que no todo se puede clasificar, por lo que el uso de campos denominados "otros" o "indeterminados" no deben avergonzar al autor de un trabajo.

- Al publicar utilizando un sistema de cuantificación -o varios- siempre es positivo que se describa con detalle la técnica utilizada, de modo que se pueda distinguir exactamente de otras semejantes, como puede suceder con el NMI -que puede ser ponderado o no- para saber si esos resultados pueden ser contrastados con otros de semejante naturaleza.

- A pesar de que la legislación de algunas comunidades autónomas permite purgar materiales arqueológicos -siempre bajo justificación y permiso administrativo-, entendemos que al nivel de conocimiento con el que en la actualidad contamos sería necesario no deshacerse ni siquiera de los fragmentos amorfos o informes, ya que las pastas y arcillas pueden proporcionar informaciones que hasta hace apenas una década eran prácticamente desechables, como procedencia, tecnología o contenidos, convirtiéndose este tipo de fragmentos no diagnosticables en elementos particularmente válidos para estos y otros tipos de muestras que en el futuro pudieran desarrollarse. Ni que decir tiene que debe recordarse no limpiar de ninguna manera aquellos ítems que se consideren adecuados para ciertas analíticas, particularmente en el caso de los contenidos.

- En cuanto a la representación gráfica de resultados de estudios de conjuntos, y siguiendo criterios de calidad visual y facilidad de acceso a la información, resulta interesante utilizar los gráficos de barras o de columnas, evitando, en la medida de las posibilidades, los diagramas de áreas o los de sectores, pues son mucho menos visuales, especialmente cuando se cuenta con un elevado número de variables.

- Dada la naturaleza de este protocolo, que intenta definir parámetros de comportamientos generales sobre materiales procedentes de contextos arqueológicos, no puede establecerse ninguna relación con el proceso de registro del material en campo, si bien se aconseja que, independientemente de la documentación específica de cada objeto, éstos siempre queden integrados dentro de algún sistema que permita identificar unidades de recogida -mínimas de excavación, alzadas, unidades estratigráficas, dependiendo de los protocolos de campo de cada equipo- y en los cuales se agrupen conjuntos de materiales que sean susceptibles de ser analizados con este protocolo. Las piezas aisladas de contextos arqueológicos - unidades de trabajo- no pueden someterse a estos protocolos al haber sido descontextualizadas.

- La descripción de pastas y los análisis arqueométricos quedan fuera de los parámetros del protocolo, que busca sistematizar un registro que facilite la accesibilidad a la mayor cantidad de datos posibles, planteando modelos de uso rápido, flexible, versátil y con la menor complejidad posible. Los resultados de cualquier tipo de analítica -pastas, contenidos, termoluminiscencia...- o temas específicos como sistemas decorativos quedan dentro de otros ámbitos no cuantificables, por lo que formando parte del discurso no se reflejan en este protocolo, entre otras cosas, porque son mucho más infrecuentes de lo que sería deseable.

- Una de las principales ventajas del protocolo es que permite gestionar un elevado número de piezas en un tiempo muy reducido. La experiencia en el yacimiento francés de Lattes demuestra que con un equipo adecuado pueden incorporarse más de 200.000 fragmentos de cerámica al mes en una base de datos modelo sirA, donde la mayor parte de los campos están automatizados y garantizan además la casi total eliminación de errores por uso exagerado de códigos de identificación y ventanas de autoentrada de datos; las bibliotecas de ayuda permiten una rápida identificación, e irían mejorando conforme se vaya ampliando su uso por parte de la comunidad científica, ya que su filosofía es de freeware de aportación canalizada por un coordinador 
único. Es cierto que se requiere personal especializado en las diversas fases de ocupación del yacimiento, pero en todo caso eso es propio de un equipo transdisciplinar actualizado. En última instancia insistimos que pueden honestamente utilizarse los campos de indeterminado, de modo que al menos no se clasifiquen erróneamente ciertas piezas y queden incorporadas en cuantificaciones que tendrían un escaso valor representativo.

Cualquier protocolo es una forma ideal de organizar la información -bases de datos-, pero la realidad cotidiana crea una serie de dificultades de las cuales somos conscientes. No siempre los arqueólogos son capaces de identificar una forma o una pasta cerámica, por lo que es preferible asignar provisionalmente un nombre nuevo relacionado con el yacimiento -por ejemplo Colomina A, UE-128-001a la espera de que otros investigadores establezcan una equivalencia. Por supuesto, pueden existir también errores de atribución a una determinada que no podrán ser detectados hasta que alguien vuelva a revisar el conjunto. Nuestro protocolo intenta mejorar la práctica de registro y cuantificación de cerámica arqueológica, si bien entendemos que puede ser susceptible de mejora en el futuro.

\section{Bibliografía}

Adroher, A. M.; Sánchez, A. y De la Torre, I. (2015): "Cuantificación en cerámica, ¿ejercicio especulativo o ejercicio hipotético? Las cerámicas ibéricas y púnicas en la Iliberri del siglo iv a.C. procedentes del depósito de la calle Zacatín (Granada)", Archivo Español de Arqueología, 88, pp. 39-65.

AlarCÃo, J. (1976): "Les amphores". En AlarCÃo, J. y Etienne, R. (dirs.): Fouilles de Conimbriga, VI. Céramiques diverses et verres. Paris: Diff. E. de Boccard, pp. 79-91.

Anselmino, L.; Coletti, C. M.; Ferranti, M. L. y Panella, C. (1986): "Ostia. Terme del Nuotatore". En Giardina, A. (dir.): Società romana e impero tardoaantigo, III. Le merci gli insediamenti. Roma: Laterza, pp. 45-81.

(C) Universidad de Salamanca
AquiLuÉ, X. (1987): Las cerámicas africanas de la ciudad romana de Baetulo (Hispania Tarraconensis). BAR Int. Series, 337. Oxford: Archaeopress.

AquiluÉ, X. y Roca, M. (eds.) (1995): Ceràmica comuna romana d'època alto-imperial a la Península Ibèrica: estat de la qüestió. Monografies emporitanes, 8.

Arcelin, P. y Tuffreau-Libre, M. (eds.) (1998): La quantification des céramiques: conditions et protocole: actes de la table ronde du Centre archéologique européen du Mont-Beuvray, Glux-en-Glenne. Bibactre, 2. Bibracte: Centre archéologique européen du Mont Beuvray.

Beltrán Lloris, M. (1998): El Instrumentum Domesticum de la 'Casa de los Delfines' - Colonia Victrix Iulia Lepida-Celsa (Velilla de Ebro, Zaragoza). Zaragoza: Institución Fernando el Católico.

Beltrán Lloris, M. (2001): “Ánforas béticas en la Tarraconense: bases para una síntesis". En Congreso Internacional Ex Baetica amphorae, Conservas, aceite y vino de la Bética en el Imperio Romano (Sevilla-Écija, 1998). Écija: Editorial Gráficas Sol, vol. II, pp. 441-537.

Bes, Ph. y Poвlome, J. (2008): "(Not) see the wood for the trees? $19,700+$ sherds of sigillata and what we can do with them", Rei Cretariae Romanae Fautores Acta, 40, pp. 505-514.

Bourgeors, A. y Mayet, F. 1991: "Les sigillées". En Fouilles de Belo, VI. Publications de la Casa de Velázquez, 14. Paris.

Carignani, A.; Ciotola, A.; Pacetti, F. y Panella, C. (1986): "Roma. Il contesto del Tempio della Magna Mater sul Palatino". En Giardina, A. (dir.): Società romana e impero tardoantico. III. Le merci, gli insediamenti. Roma: Laterza, pp. 27-43.

Carreras, C. (2000): Economía de la Britannia romana: la importación de alimentos. Barcelona.

Carreras, C. (2001): "Ánforas de la Campa Torres (Campañas 1985-1997)”. En Maya, J. L. y CuesTA, F. (eds.): El castro de La Campa Torres. Gijón, pp. 389-392.

Carreras, C. y Morais, R. (eds.) (2011): Ánforas romanas de Lugo. Comercio romano en el Finis Terrae. Traballos de Arqueoloxía, 3. Lugo.

Carvalho, T. P. (1998): A Terra sigillata de Monte Mozinho: contributo para a história económica do povoado. Cadernos do Museu de Penafiel, 3. Penafiel: Museu Municipal.

Coelho-Soares, A. y Silva, C. T. DA 1978: "Ânforas romanas da área urbana de Setúbal”, Setúbal Arqueológica, IV, pp. 171-201. 
Corremas, M.; Poblome, J.; Bes, P. y Waelkens, M. (2010): "The quantification of amphorae from Roman Sagalassos, Southwest Turkey”. En Horejs, B.; Jung, R. y Pavúk, P. (eds.): Analysing pottery. Processing, Classification and Publication, Studia Archaeologica et Medievalia, x. Bratislava, pp. 285-303.

Delgado, M.; Mayet, F. y Alarcão, A. M. (1975): Les sigillées. Fouilles de Conimbriga, Iv. Paris.

Escrivì, V. (1989): La cerámica romana de Valentia: la terra sigillata hispánica. Serie Arqueológica Municipal, 8. Valencia.

Fernández Fernández, A. (2014): El comercio tardoantiguo (ss. IV-VII) en el Noroeste peninsular a través del registro cerámico de la Ría de Vigo. Roman and Late Antique Mediterranean Pottery, 5. Oxford.

Fernández Izquierdo, A. (1984): Las ánforas romanas de Valentia y de su entorno maritimo. Ayto. de Valencia.

García Vargas, E. (2007): "Hispalis como centro de consumo desde época tardorrepublicana a la antigüedad tardía. El testimonio de las ánforas", Anales de Arqueología Cordobesa, pp. 317-360.

Gonçalves, V. S. (1971): O Castro da Rotura e o vaso campaniforme. Setúbal: Junta Distrital.

Hayes, J. W. (1976): "Pottery: Stratified Groups and Typology". En Humphrey, J. H. (dir.): Excavations at Carthage 1975 conducted by the University of Michigan, I. Túnez: Cérès, pp. 47-123.

Hayes, J. W. (1978): "Selected deposits (continued)". En Humphrey, J. H. (dir.): Excavations at Carthage 1975 conducted by the University of Michigan. Ann Arbor: Kelsey Museum, vol. II, pp. 113-118.

KeaY, S. (1984): Late Roman amphore in the western Mediterranean. A tipology and economic study: the Catalan evidence. BAR Int. Series, 196. Oxford: Archaeopress.

Keay, S. J. (1998): "African amphorae". En Saguí, L. (dir.): Ceramica in Italia: VI-VII secolo. Atti del Convegno in onore di John W. Hayes. Firenze, pp. 141-155.

Lopes, C. (1994): A sigillata de Represas. Tratamento informático. Coimbra: Fac. de Letras de Coimbra-Instituto de Arqueologia.

Lopes, C. y Mayet, F. (1990): "Commerce régional et lointain des amphores lusitaniennes". En Alarcão, A. y MaYet, F. (eds.): Anforas lusitanas. Tipologia, produção, comércio/Les amphores lusitaniennes. Typologie, production, commerce (actas da mesa-redonda de Conimbriga, 1988). Conimbriga-Paris: Museu Monográfico de Conimbriga-E. de Boccard, pp. 295-303.

Marlière, E. y Torres, J. (2005): “Tonneaux et amphores à Vindolanda: contribution à la connaissance de l'approvisionnement des troupes stationnées sur le mur d'Hadrien (II)". En Birley, A. y BlaKe, J. (eds.): Vindolanda. The excavations of 2003/2004. Durham, pp. 214-236.

Mateo, D. y Molina, J. (2016): “Archaeological Quantification of Pottery: The Rims Count Adjusted using the Modulus of Rupture (MR)", Archaeometry, 58, pp. 333-346.

DOI: $10.1111 /$ arcm. 12171.

Millet, M. (1991): "Pottery: population or suppply patterns?”. En BAKER, G. y LloYd, J. (eds.): Roman landscapes. London, pp. 18-26.

Molina, J. (1997): La dinámica comercial romana entre Italia e Hispania Citerior (siglos II a. C.- II d. C.). Alicante.

Montesinos i Martínez, J. (1991): Terra sigillata en Saguntum y tierras valencianas. Valencia: Caja de Ahorros de Sagunto.

MoraIs, R. (1998): As ânforas da zona das Carvalheiras: contribuição par o estudo das ânforas romanas de Bracara Augusta, Braga. Cadernos de Arqueologia (Braga, Portugal). Monografias, 8. Braga.

Morais, R. (2005): Autarcia e comércio em Bracara Augusta. Contributo para o estudo económico da cidade no periodo Alto-Imperial. Bracara Augusta. Escavaçóes Arqueológicas, 2. Braga.

Orton, C. (1982): "Computer simulation experiments to assess the performance of measures of quantity of pottery", World Archaeology, 14, pp. 1-20.

Orton, C.; Tyers, P. y Vince, A. (1993): Pottery in archaeology. Cambridge.

Pascual, G. (1998): "Los materiales tardorrepublicanos del yacimiento submarino de Pudrimel (La Manga del Mar Menor, Murcia)". En Puertos antiguos y comercio maritimo. III Jornadas de arqueología subacuática (Valencia, 1997). Valencia, pp. 263-289.

Peacock, D. P. S. y Williams, D. F. (1986): Amphorae and the Roman economy. An Introductory Guide. London: Longman.

Pellicer, M. (1978): “Tipología y cronología de las ánforas prerromanas del Guadalquivir, según el Cerro Macareno (Sevilla)", Habis, 9, pp. 365-400.

Picon, M. (1975): "Céramique antique et détermination des provenances", Dossiers de l'Archéologie Française, 9, pp. 83-93.

Polak, M. (2000): South Gaulish Terra sigillata with potter's stamps from Vechten. Rei Cretariae Romanae Fautorum Acta, Suppl., 9. Nijmegen.

Pollard, R. (1990): "Quantification: Towards a standard practice", Journal for Roman Pottery Studies, 3, pp. 75-79. 
Quaresma, J. C. (2012): Economia antiga a partir de um centro de consumo lusitano. Terra sigillata e cerâmica africana de cozinha em Chãos Salgados (Mirobriga?). Estudos e Memórias, 4. Lisboa: UNIARQ.

Ramón, J. (1995): Las Ánforas Fenicio-Púnicas del Mediterráneo Central y Occidental. Col.lecció Instrumenta, 2. Barcelona.

Remolà, J. A. (2000): Las ánforas tardo-antiguas en Tarraco: (Hispania Tarraconensis). Siglos IV-VII d.C. Col-lecció Instrumenta, 7. Barcelona.

Reynolds, P. (2010): Hispania and the Roman Mediterranean, $A D$ 100-700. Ceramics and Trade. London: Duckworth.

Rice, P. M. (1987): Pottery Analysis. A source book. Chicago.

Riley, J. A. (1976): "The Carthage system for the quantification of pottery". En Humphrey, J. H. (dir.): Excavations at Carthage 1975 conducted by the University of Michigan, I. Túnez: Cérès, pp. 125-131.

Shennan, S. (1992): Arqueología cuantitativa. Barcelona: Crítica.

Silva, C. T.; Coelho-Soares, A. y Correia, V. (1990): "Produção de Ânforas romanas no Martinhal (Sagres)”. En Alarcão, A. y Mayet, F. (eds.): Anforas lusitanas. Tipologia, produção, comércio/Les amphores lusitaniennes. Typologie, production, commerce (Actas da mesa-redonda de Conímbriga, 1988). Conimbriga-Paris: Museu Monográfico de Conimbriga-E. de Boccard, pp. 225-246.

Silva, C. T.; Soares, J.; Beirấo, C. M.; Dias, L. F. y Coelho-Soares, A. (1980-1981): "Escavaçóes arqueológicas no Castelo de Alcácer do Sal (campanha de 1979)", Setúbal Arqueológica, 6-7, pp. 149-218.

Silva, R. C.; Fernández, A. y Carvalho, P. (2015): "Contextos e cerâmicas tardo-antigas do fórum de Aeminium (Coimbra)", Revista Portuguesa de Arqueologia, 18, pp. 237-256.

TED'a (1989): Un abocador del segle $v$ d.C. en el fórum provincial de Tàrraco. Memóries d'Excavació, 2. Tarragona.

Tomber, R. (1989): "Pottery from the 1982-1983 excavations". En Humphrey, J. H. (dir.): The circus and a byzantine cemetery at Carthague. Ann Arbor: Kelsey Museum, vol. I, pp. 437-528.

Tomber, R. (1993): "Quantitative approaches to the investigation of long-distance exchange", Journal of Roman Archaeology, 6, pp. 142-166.

Verdan, S.; Theurillat, Th. y Kenzelmann, A. (eds.) (2011): Iron Age pottery: a quantitative approach. Round Table, Athens (2008). BAR Int. Ser., 2254. Oxford: Archaeopress.

Viegas, C. (2003): Terra sigillata da Alcáçova de Santarém. Economia, comércio e cerâmica. Trabalhos de Arqueologia, 26. Lisboa: Instituto Português de Arqueologia.

Viegas, C. (2011): A ocupação romana do Algarve. Estudo do povoamento e economia do Algarve central e oriental no periodo romano. Estudos e Memorias, 3. Lisboa.

Whitehouse, D.; Costantini, L.; Guidobaldi, F.; Passi, S.; Pensabene, P.; Pratt, S.; Reece, R. y Reese, D. (1985): "The Schola Praeconum II", Papers of the Bristish School at Rome, vol. LIII, pp. 163-210. 